University of Redlands

\title{
SNARC Data Portal Project
}

A Major Individual Project submitted in partial satisfaction of the requirements for the degree of Master of Science in Geographic Information Systems

\author{
by \\ Zheming Sun \\ Douglas M. Flewelling, Ph.D., Committee Chair \\ Ruijin Ma, Ph.D.
}

August 2020 
SNARC Data Portal Project

Copyright (C) 2020

by

Zheming Sun 
The report of Zheming Sun is approved.

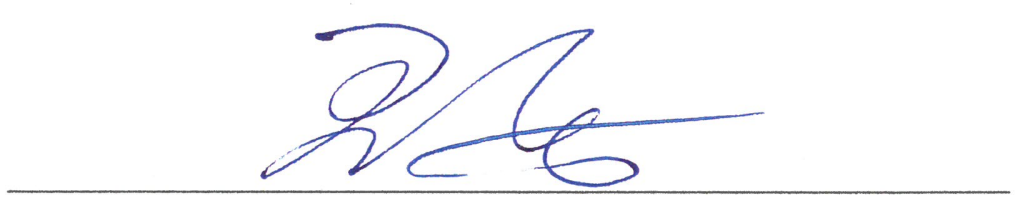

Ruijin Ma, Ph.D.

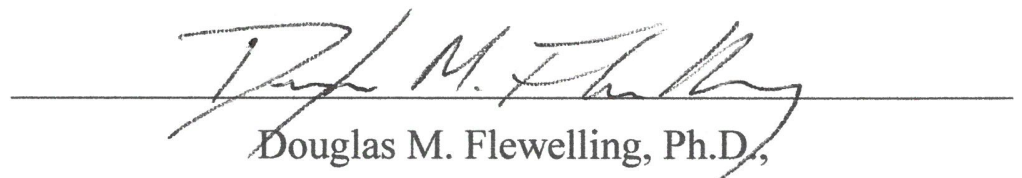

August 2020 



\section{Acknowledgments}

This project could not be completed without several individuals. I want to say thank you for all the faculty in the Geographic Information Science department of the University of Redlands Fang Ren, Ruijin Ma, Mark Kumler, Andrea Alvarado, and especially my advisor Douglas Flewelling. I do not have any GIS background before I entered this program, Dr. Flewelling always helps me think in a GIS way and solve any problems with his profound knowledge and patient. To Cohort 36, thank you to everyone for being such a wonderful group.

Thank you to my project clint, Smith and Matthew Green, from SNARC, for helping me finish this project. 



\section{Abstract \\ SNARC Data Portal Project \\ by \\ Zheming Sun}

The Sierra Nevada Aquatic Research Consortium (SNARC) is an organization that focuses on aquatic studies, including stream hydrology and ecology, around the Sierra Nevada Area between the Central Valley of California and the Great Basin. As the research progresses, more datasets have been generated by the researchers. Store and find the correct data-efficient become a problem in their organization. This paper provides a solution to this problem.

This paper will introduce how to create a data portal that research in SNARC can upload, access, and share the datasets. A stand-alone geoprocessing tool that can analyze the uploaded data and find the geographic relationship will be in the deploy in the data portal. The data portal and geoprocessing tools, including ArcGIS Online, ArcGIS JavaScript API, GIS fundamental knowledge, Python, and Django web platform, are collaborated to conduct the analyst. The final deliverable of this project will be a website. The dataset analysis tools will run automatically when the user uploads the new dataset. 



\section{Table of Contents}

Chapter 1 - Introduction .......................................................................................... 1

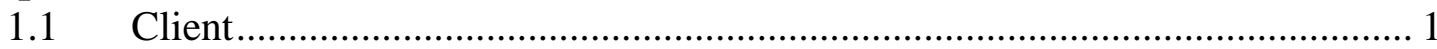

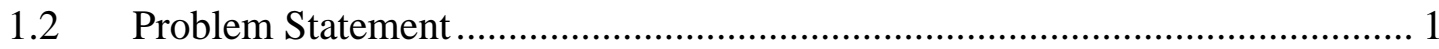

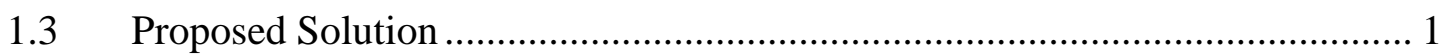

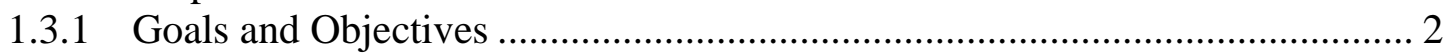

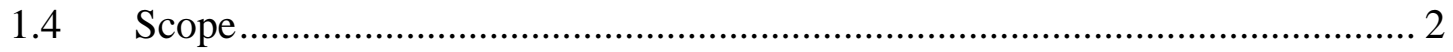

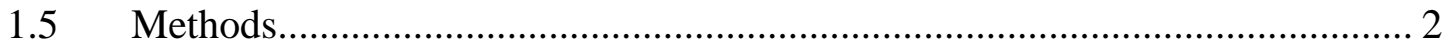

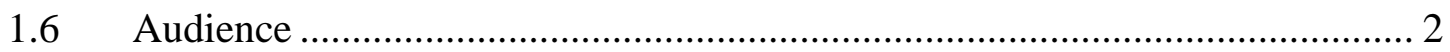

1.7 Overview of the Rest of this Report .......................................................... 2

Chapter 2 - Background and Literature Review ............................................................... 5

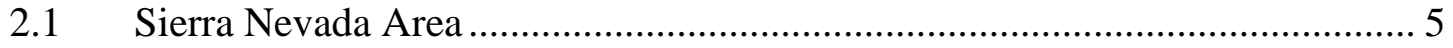

2.2 Indexing with Tree Structure …………………................................... 8

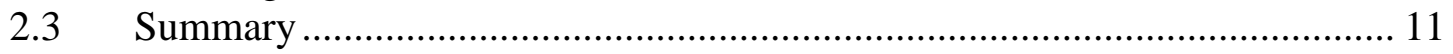

Chapter 3 - Systems Analysis and Design.......................................................................... 12

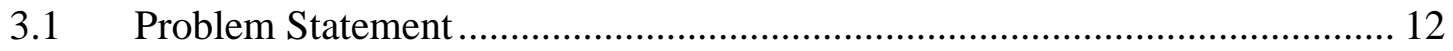

3.2 Requirements Analysis …………………………............................. 12

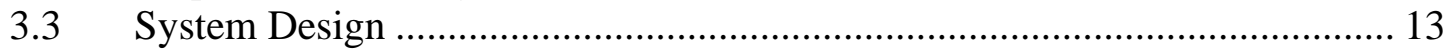

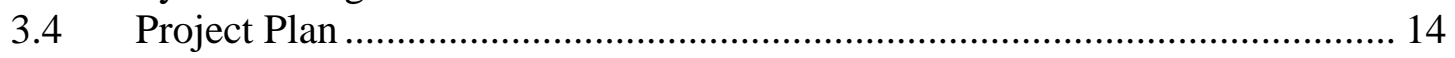

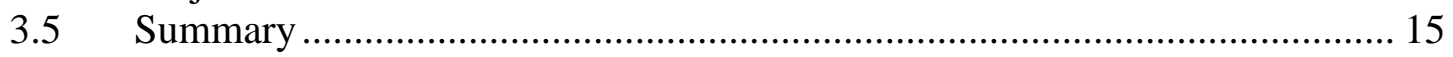

Chapter 4 - Database Design...................................................................................................... 17

4.1 Conceptual Data Model ...................................................................... 17

4.2 Logical Data Model ........................................................................ 18

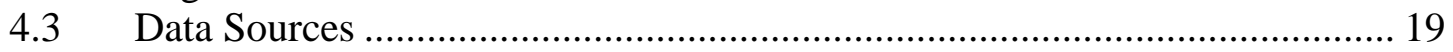

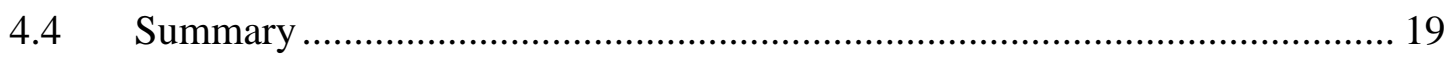

Chapter 5 - Implementation......................................................................................... 21

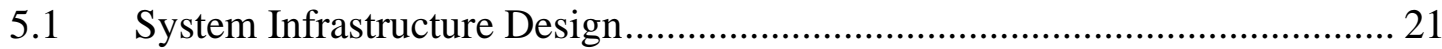

5.2 Build a web application with Django........................................................... 22

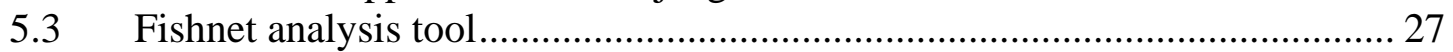

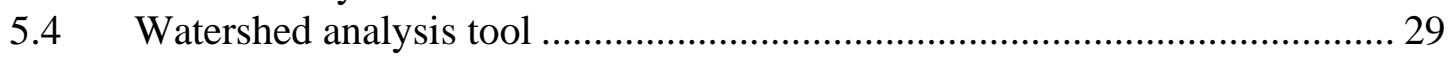

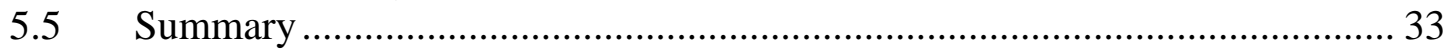

Chapter 6 - Results and Analysis....................................................................................... 35

$6.1 \quad$ Result and Analysis of the SNARC Datasets ................................................ 35

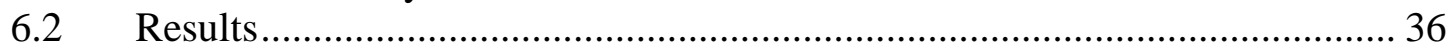

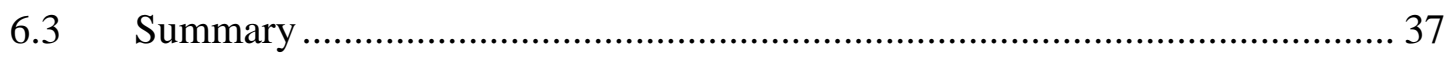

Chapter 7 - Conclusions and Future Work ................................................................... 39

$7.1 \quad$ Summary and Conclusion ......................................................................... 39

7.2 Hydrological Analysis System.................................................................. 39

Works Cited 41 
Appendix A. SQL Database Design............................................................................. 43

Appendix B. Analysis tools ...................................................................................................... 44

Appendix C. Django …......................................................................................................... 47 


\section{Table of Figures}

Figure 2-1: Boundary of the Sierra Nevada Area ......................................................... 6

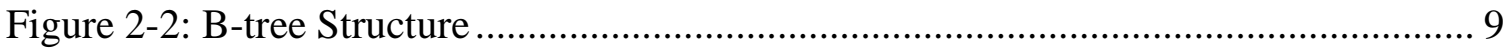

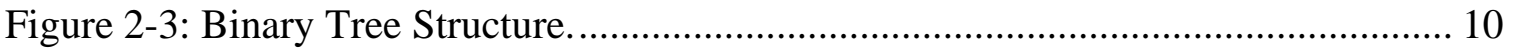

Figure 2-4: Sample of a Valid Binary Search Tree........................................................ 11

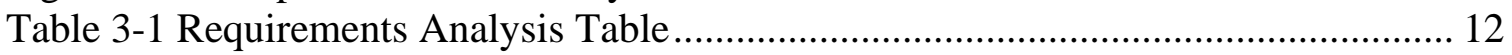

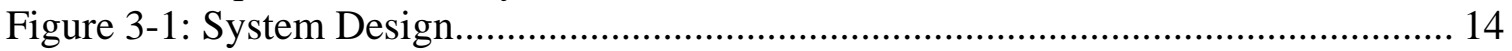

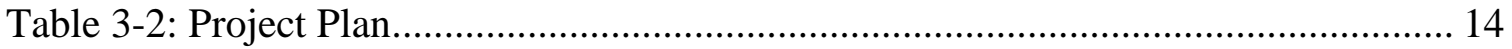

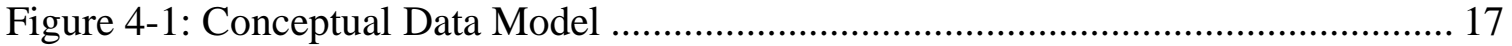

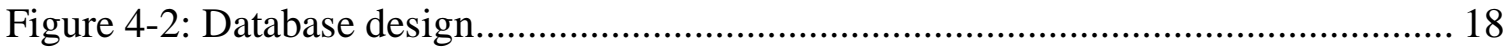

Figure 5-1: Technical Stack in the Linux server........................................................ 21

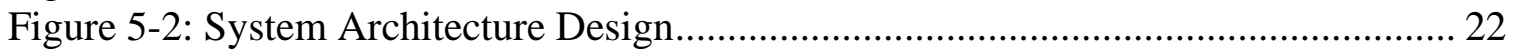

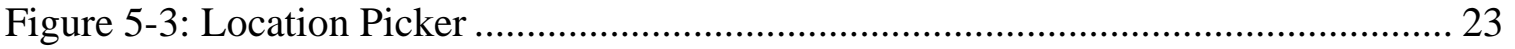

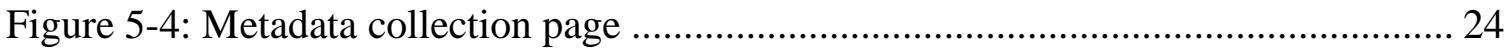

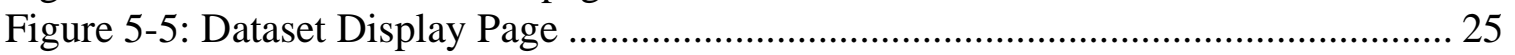

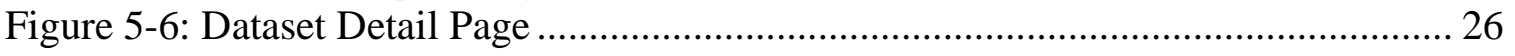

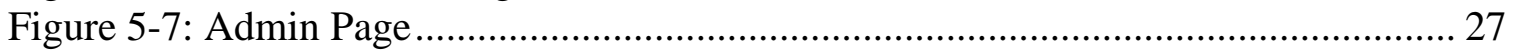

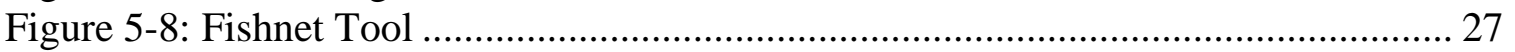

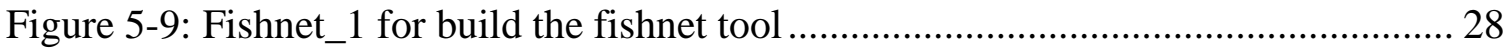

Figure 5-10: Relationship Between Fishnet_1 and Fishnet_2 .................................... 29

Figure 5-11: Watershed boundary with ID HUC4. Right: HUC8 zoom to the \#1804 area

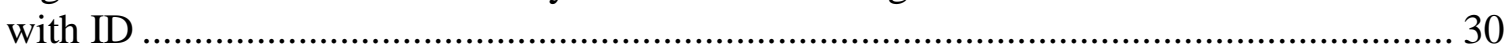

Figure 5-12: California State Boundary and USGS Watershed \#18 Boundary ................ 31

Figure 5-13: The Fishnet, States, and Watershed Boundaries........................................ 32

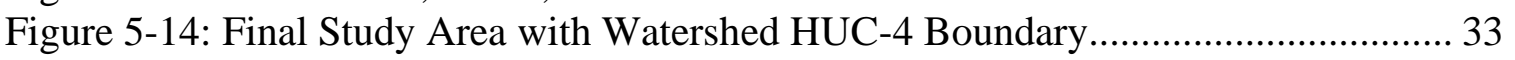

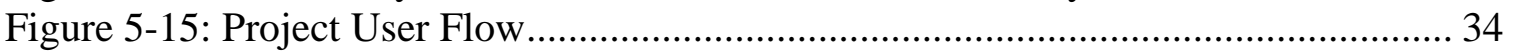

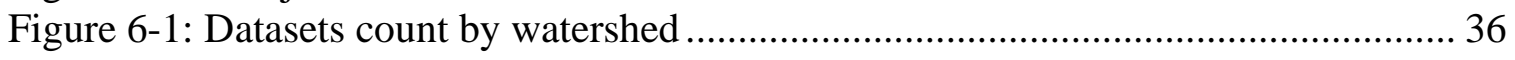

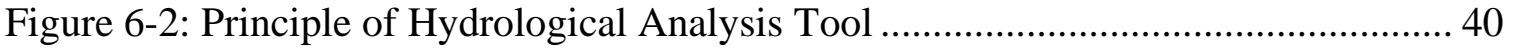




\section{List of Tables}

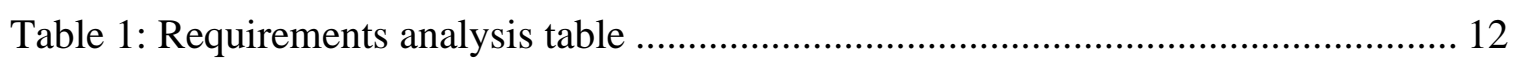

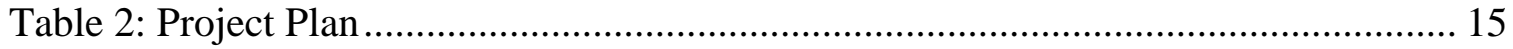




\section{List of Acronyms and Definitions}

SNARC - Sierra Nevada Aquatic Research Consortium

USGS - United States Geological Survey

MVC - Model-view-controller which is a software design pattern 



\section{Chapter 1 - Introduction}

\subsection{Client}

The Sierra Nevada Aquatic Research Consortium (SNARC) is an organization that focuses on the aquatic ecosystems of the Sierra Nevada area. Thousands of lakes and streams data in the high Sierra has been collected in the last several decades by the researchers in SNARC.

Many of these researchers work out of the Sierra Nevada Aquatic Research Laboratory (SNARL), a sizeable biological field research station operated through the University of California Natural Reserve System located in Mammoth Lakes, California. SNARL served as the central coordinating entity for this group and will serve as the home for the Data Interface Tool. Most of the researchers in SNARC are the master or $\mathrm{Ph} . \mathrm{D}$. student at the University of California system.

\subsection{Problem Statement}

Three problems were addressed during requirement collection and conversation with the SNARC. The first one was that the researchers in SNARC had collected thousands of datasets, and the existing way they manage the data is not enough for future development. Those datasets currently store in the researchers' personal computer without any indexing.

The second question is the SNARC is an organization that focuses on the aquatic system in the lakes and rivers. The researchers need a platform that can search and access the data with geographic or aquatic features.

The last problem addressed in this project is that the researchers need to extract useful information from data and to make the decision based on the data result of the analysis.

\subsection{Proposed Solution}

This project use ArcGIS and Python script to solve this problem. The first step is to build a web portal with Python and Django. The second step is to get the geological information about the datasets. The last step builds the analysis tool with Python and ArcGIS API to indexing the data.

The Django web platform can meet all the requirements for a data portal in this project, such as upload and download the file, user management system, and the modelview-controller (MVC) structure. Django was the most fitting solution for this problem.

According to the sample data from Sierra Nevada Aquatic Research Consortium, the datasets storage in several different formats (CSV, shapefile, and JSON), and not all of them have metadata. The SNARC also provides a metadata template that they want to use it in this system. A web portal developed with Python and ArcGIS will help enforce the metadata management. 
Some geoprocessing tools and Python script can be used to analyze and index the datasets. The indexing should base on the geological and aquatic information. With this indexing, the user can filter and search with the keyword such as the watershed number and waterbody connection information.

\subsubsection{Goals and Objectives}

The primary goal of this project was to help the client create a data portal to manage their geographic data. The SNARC does not have a technical department, so the technology

used in this project should be simple and easy to maintains. The larger goal of this project is to generate the report with the analysis tool to monitor the datasets in the data portal and provide the suggestion for the next move of the SNARC.

\subsection{Scope}

Consider the operation efficiency of the analysis system, the study area of this project should cover the Sierra Nevada Area in California, and the southern part of Oregon. This project avoided using the ArcGIS enterprise and ArcGIS Pro API, so the operation speed of some features in this project may not be the best solution. The last scope is the continuing maintenance issue. This project allowed the admin user to change the configuration of the web portal in the admin page. Compare with the direct control with the code, this design trade-off some system flexibility

\subsection{Methods}

To store and analyze the datasets, all the data should be stored individually, and the metadata stored in the MySQL database. The state and watershed boundaries data were downloaded from the United States Geological Survey official website and ArcGIS data portal. All the data was added to the maps in ArcGIS Pro. The fishnet data also generated by the ArcGIS pro geoprocessing tool. The fishnet and watershed data were saved in the JSON file format to avoid the delay of the response time of the ArcGIS API.

\subsection{Audience}

The audience of this paper is the GIS professional with some computer science background. The GIS professional should know the fundamental knowledge of the GIS and basic ESRI software knowledge. Computer science covered the Python and ModelView-Controller (MVC) web portal knowledge.

\subsection{Overview of the Rest of this Report}


The project report has been organized to include six more chapters. Chapter Two discusses the background of this project and the detail information of the client and study area. Chapter Three shows the system design and schedule plan. Chapter Four include the method of dataset storage and database model design. Chapter Five shows the detail of how to build the web portal and analysis tools. Chapter Six is the result of this project. 



\section{Chapter 2 - Background and Literature Review}

The client of this project aimed to research the aquatic and water system in the Sierra Nevada area. Therebefore, it was essential to develop a deep understanding of the geological and water environment of this area. Section 2.1 details that information about the Sierra Nevada Area.

Collecting and indexing the data are the final goals of this project. This project uses the most popular search tree structure, binary search tree, and B-tree to build the data index. Section 2.2 explains the detail of the search tree structure.

\subsection{Sierra Nevada Area}

The Sierra Nevada region is geologically defined from Southern to Northern California as one huge mountain along the west coast of North America. The Sierra Nevada region has been explored and continually examined over the years. Figure 2-1 shows the location of the Sierra Nevada area. 


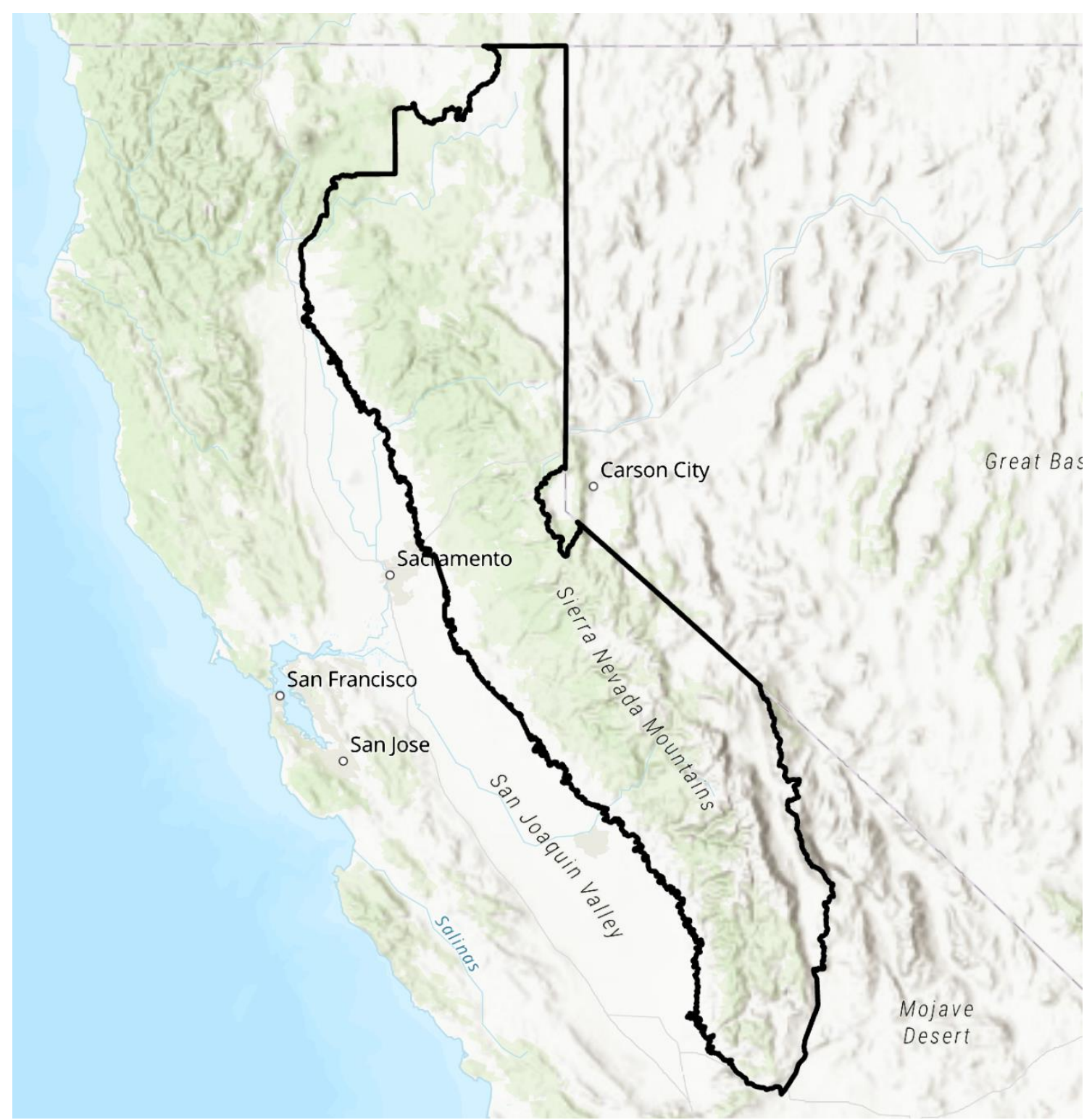

Figure 2-1: Boundary of the Sierra Nevada Area

The Sierra Nevada formed millions of years ago with the movements of the continent. The history of the Sierra Nevada can be traced back 130 million years ago, along with the movement of the sea and the cooling progress of molten granite earth (Hill, 1975). It contained the highest point in the contiguous United States: Mount Whitney (Stroh \& Shalit, 2015), as well as some famous sites including Lake Tahoe, Yosemite National Park, and Kings Canyon. Although most people get to know the Sierra Nevada from the Brewing Company, which produces tons of beer every year within the United States, it is not only a name behind the brown glass bottles. This region includes a portion of Nevada State around Lake Tahoe, sharing the same mountain range for about four hundred miles long and forty to eighty miles in breadth (Farquhar, 2007). Such identifying features of the Sierra Nevada made it one of the most significant places 
to conduct research and educational activities. Abundant research has been conducted within the Sierra Nevada range as that its mountains are covered with unique rocks, forests, weather, watersheds, and wildlife. Tons of researchers, and organizations that came here to do the independent research.

Among all the unique geological and geographical features of the Sierra Nevada region, water has been outstanding and deeply studied from aspects of water flow, lakes, and creatures inside. Water appeared here as early as rocks to the Sierra Nevada in forms of ice and cloud with the movements of glaciers, tracing back to the Ice Age (Hill, 1975). These water sources then melted into running water, streams, and lake water, running continually as a source of further development of the current landscapes of California, and feeding the plants and creatures in between. The aquatic systems are one of the most popular study areas around the Sierra Nevada region.

To conduct research focusing on the Sierra Nevada aquatic ecosystems ranging from cold snow to warm streams, many previous works can be used as an essential reference. There are several aspects of research data that can be used from earlier studies. Earlier datasets can be categorized from the studies that described experiments and focused surveys that have been conducted on specific species, communities, sites, or ecosystem processes. The scope of such studies in geographic scales and temporal scales.

To separate the effects from other environmental and chemical factors, the geological distribution changing throughout the years has been one crucial feature used in the previous studies. The earlier studies gathered data from short-term or long-term scales of time within specific historical frames. Time scale over history is one of the critical research criteria, divided into decades, years, months, even daytimes (Leland, Fend, \& Mahood, 1986). A typical template for research time can be found around target areas. The temperature and climate-changing from the previous decade was used to compare the behaviors of existing ecosystems in the Sierra Nevada, as well as future predictions (Viers, 2011).

From the geographic division, comparisons can be made between lakes all around the Sierra Nevada areas. Over the last several decades, the different leveled data have been collected on thousands of lakes and streams in the high Sierra. Several explicitly ecological datasets described the community composition of lakes, ponds, and streams, mainly for zooplankton and aquatic insects (Knapp, Non-native trout in natural lakes of the Sierra Nevada: an analysis of their distribution and impacts on native aquatic biota, 1996). To limit the study area, boundaries are necessary for the Sierra Nevada elevation distribution of fishing (Leland, Fend, \& Mahood, 1986).

Some datasets focus on physical and chemical processes, including decades' oxidation data, which influenced the geothermal region from upstream to downstream (Wilkie \& Hering, 1998). Diverse datasets have the potential to complement each other and inform new research and resources, while similar datasets exist for Sierra streams. In contrast to the research effort that has been devoted to quantifying the impact of introduced trout on native lake biota, few studies have examined their effect on native stream biota (Knapp, Matthews, \& Sarnelle, Resistance and resilience of alpine lake fauna to fish introductions, 2001). The findings drew attention to the necessities of the sorting of chemical factors. 
As one of the widely used research targets, native and non-native trout behaviors were observed and collected (Herbst, Silldorff, \& Cooper, 2009). From earlier work, this affected the distribution of a wide range of native aquatic species in the Sierra Nevada, including native fishes, amphibians, zooplankton, and benthic macroinvertebrates (Knapp, Non-native trout in natural lakes of the Sierra Nevada: an analysis of their distribution and impacts on native aquatic biota, 1996). Taking one species of fish as an example can narrow down the targets and focus on one specific data from the massive dataset.

To analyze the date, different variables, like density, size, level, population, and even the flows, were included to conduct the analysis. The dataset materials were not limited to the observed facts, and the researchers collected the historical data, but the methods of geographic information system skills should be used to analyze and sort such datasets for the public. The geometric analysis should have been done to convert to readable, accessible results for those works.

Several explicitly ecological datasets described the community composition of lakes, ponds, and streams, mainly for zooplankton and aquatic insects. Other datasets focused on physical and chemical processes, including decades-long limnological (e.g., Tahoe, Castle Lake, Emerald Lake) and stream studies that describe temperature, chemistry, and flow. In addition, many small-scale experiments and focused surveys have been done on specific species, communities, sites, or ecosystem processes, and this complement and inform the larger scale, longer-term studies.

\subsection{Indexing with Tree Structure}

A database index is a data structure that improves the speed of data retrieval operations. The database indexing has the same feature as the book index, which is a key to locating the information contained in a database. Indexing is not only used in books, but it is also widely used in modern society. People around the world will generate about 44 zettabytes of data every day in 2020 (Desjardins, 2019). Usually, the personal computer has a one terabyte hard drive, and 44 zettabytes equal to 44,000,000,000 terabytes. For storing and managing big data, database indexing is indispensable.

Indexing of the relational database is a data structure that improves the speed of data retrieval operations. Indexing is related to specific tables and consists of one or more keys. For example, if the reader wants to find the specific paragraph in a book without an index, they can read the book page by page until they find the paragraph. With an index, the reader can find the chapter in the index and just read the chapter to find that paragraph. Database indexing is similar to book indexing; when new data comes in, all field data will store in a clustered index, containing a key value and a pointer to the data rows. The clustered index is sorted, so when the users run the searching query, the database does not need to iterate all rows. The most popular way to index the relational database is B-tree, which is a self-balancing tree data structure.

The B-tree is a balanced search tree. It was invented by Rudolf Bayer and Edward M. McCreight in 1970. The B-tree was initially invented for sorting data structures stored on the hard disk. Compared with a binary tree, the length of the path from the root to the leaf is shorter. Figure 2-3 shows the basic structure of the B-Tree. This characteristic makes B-tree have less disk I/O when searching. Nowadays, the RAM of the commercial server is large enough to save the whole table in the memory; so, the modern database 
management system such as MySQL and PostgreSQL move the B-tree calculation into the memory. The B-tree must meet the following characteristics:

1. All paths from the root to a leaf have the same length.

2. If the node has $n$ children, it contains $n-1$ key.

3. All the children nodes are at least half full.

4. The root has at least two children if it is not a leaf.

5. The elements stored in a given subtree all have keys that are between the keys in the parent node on either side of the subtree pointer. root node

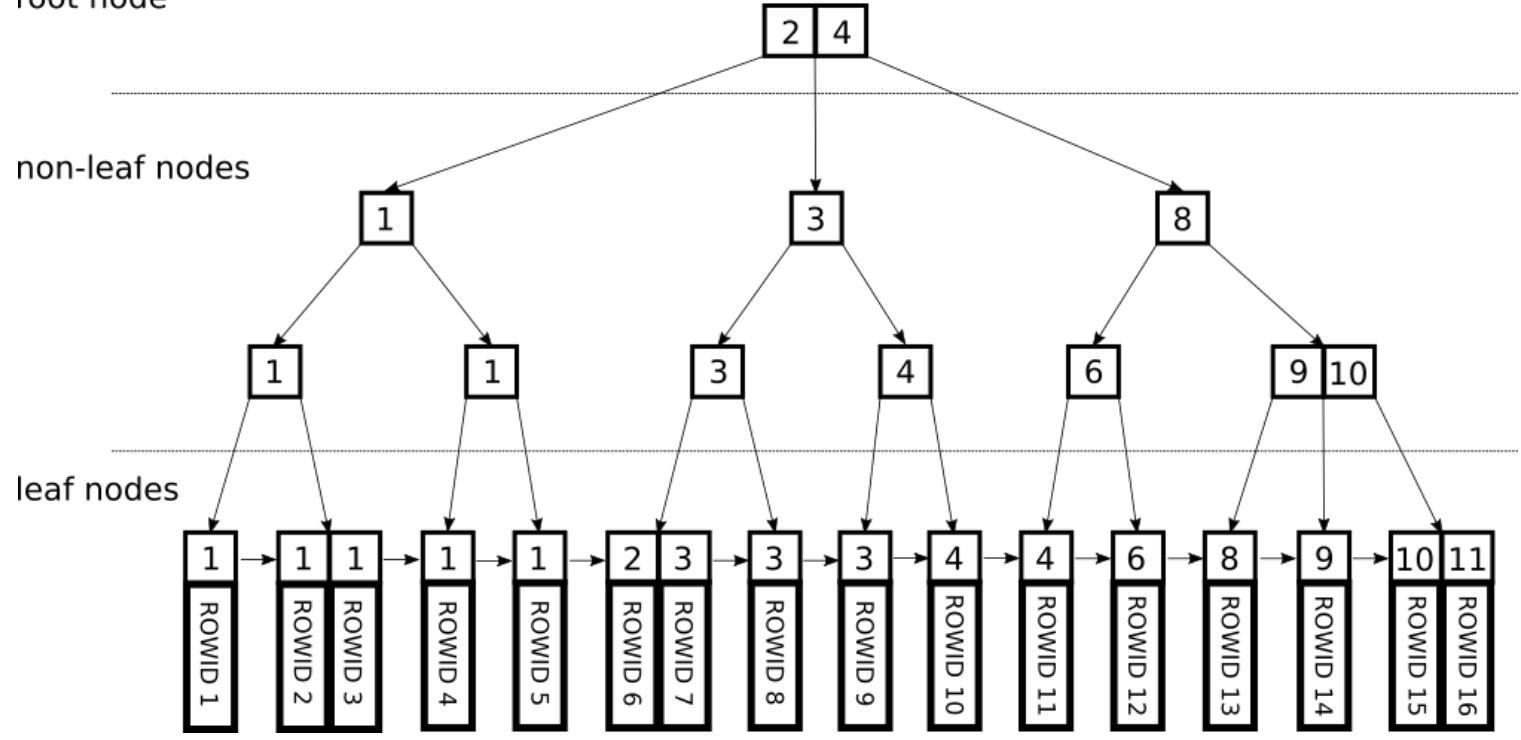

Figure 2-2: B-tree Structure

Due to the length from the root to all leaves are the same in the B-tree, the time complexity of B-tree is $\mathrm{O}(\log 2(\mathrm{n}))$. For example, if the database has 1,000,000 records, the time complexity of this database is $\mathrm{O}(\log 2(1,000,000)=19.931$. Compared with nonindex data, efficiency is raised more than 50000 times.

Another tree structure used in this project is the binary search tree. Binary Search Tree is a binary tree that follows some specific properties. Binary Tree is a tree whose elements have at most two children. The first element call root node, the node connects with the node called the left and right child. Each node in the tree contains some data. Figure 2-4 shows the basic structure and name of each part in the binary tree. 


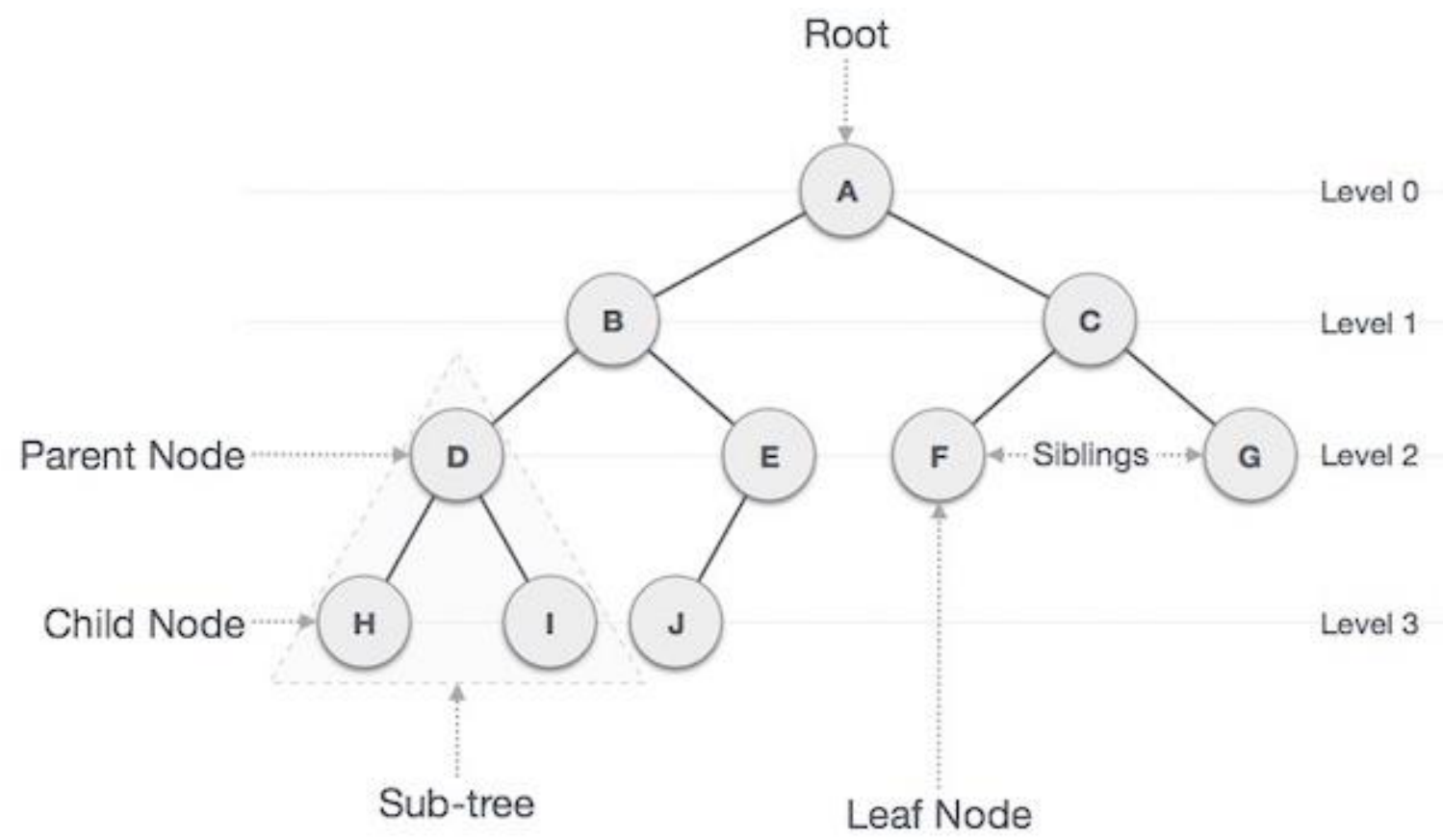

Figure 2-3: Binary Tree Structure.

Unlike the array and linked list, which are the linear structure, the binary tree is a hierarchical structure. This structure has a better operation speed when doing the insert, delete, and search action (Frost, 1982). However, only the binary tree cannot improve the operation speed. The data in the binary tree should follow some order. The binary search tree is the best choice to build an index for data.

The binary search tree is a kind of binary tree; it follows all the rule of the binary tree and has the following properties:

1. The key value in the left subtree node is always less than the key node of this node.

2. The key value in the right subtree node is always higher than the key value of this node.

Following those properties below, the binary search tree should be a sorted tree structure. With this structure, the searching becomes very simple. The searching method will compare the target value and node value if the target value is less than the node value; the next step is to go through the left node. Otherwise, it will iterate the right node. Repeat this action until the script finds the target value in the binary search tree or reach the last subtree node in the tree. The maximum path in this algorithm is the length from the root to the leaf. In most cases, the operation time of the search binary is less than any kind of search if the data were stored in an array. 


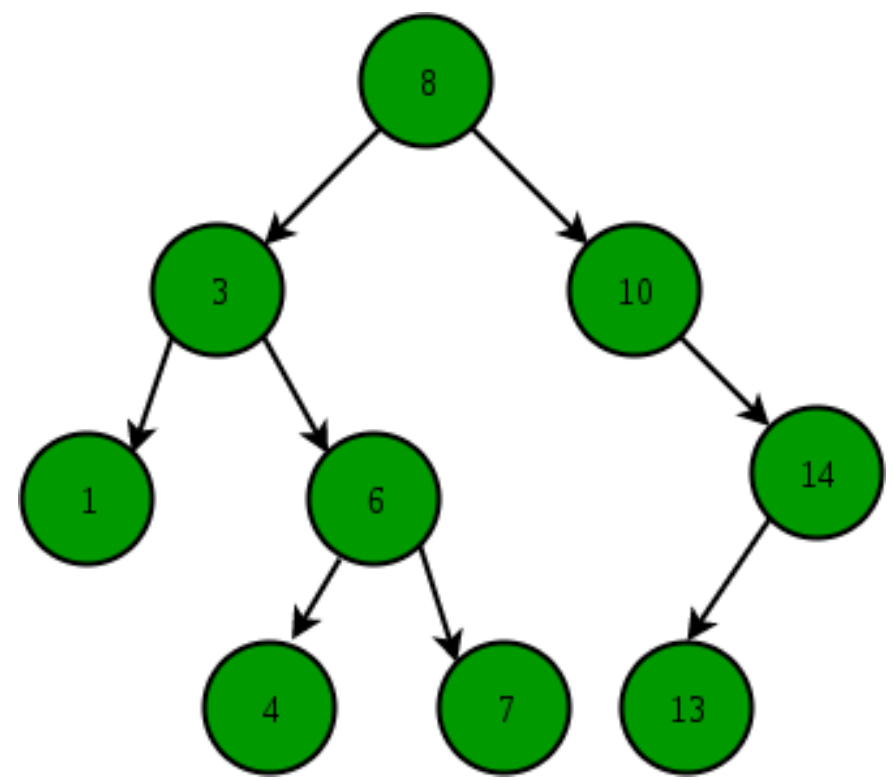

Figure 2-4: Sample of a Valid Binary Search Tree

\subsection{Summary}

This chapter introduced the necessary geological information of the Sierra Nevada Area. This chapter also addressed the three different tree structures, B-tree, binary tree, and quadtree, which are used to index the datasets. The indexing can help the researchers organize their data in the data portal. The next chapter will talk about the requirements for the system analysis. 


\section{Chapter 3 - Systems Analysis and Design}

The Chapter three will detail the design of the web portal and analysis tool. Section 3.1 will discuss the problem from the client. Section 3.2 describes the project requirements. Section 3.3 will focus on overall system design. Section 3.4 introduces the development schedule and timeline of this project. The last section 3.5 will summarize the system design and analysis.

\subsection{Problem Statement}

The researchers in SNARC need a platform to manage their data. There are four different problems addressed in this project:

1. The researchers generated lots of data in past research. Those data are currently stored on their personal computer.

2. There is no index of any data; the researchers do not know what data other researchers have. The only way they can find the data is to email everyone in the organization.

3. When someone gets a dataset from another researcher, he may edit the data and make the same data that has two different versions.

4. Sometimes the data is more than one gigabyte. The large data cannot send with an email, place them in the cloud storage need to face a lot of permission problem.

\subsection{Requirements Analysis}

The requirements from the SNARC are listed in Table 3-1.

Table 3-1 Requirements Analysis Table

\section{Requirement name}

Data upload

Validation check

USGS watershed number integration

Filter and search data

\section{Requirement description}

All the data which belong to SNARC need to be uploaded to a platform.

All the uploaded data need to pass a validation check. The validation check includes the data integrity check and data accuracy check.

The researchers in the SNARC uses the USGS watershed number to search the dataset. So, after the dataset upload to the platform, an analysis tool needs to find which watershed this dataset is in.

Use the tag and keyword to filter and search the data in the data portal. The user can define the tag, and the keyword are 
a part of metadata, the search query can get the data with the keyword.

Download the dataset Download the dataset from the data portal. All the data should be stored in the cloud storage and can download with the data portal authentication.

\subsection{System Design}

Based on the client requirements, this system should be stand-alone and easy to maintain. As one of the fastest-growing programming languages, Python is a general-purpose, high-level, and widely used programming language in recent times (Srinath, 2017). Meanwhile, the ArcGIS Pro and ArcGIS API also have excellent support for the Python.

Due to the final deliverable in this project is a web application, building a web application from zero is possible, but it takes such a long time. The open-source platform can solve this problem entirely. There are two most popular web platforms for Python, one is Flask, and another one is Django. In this case, Django has the build-in user control system, and the admin system can meet the user requirements better. Django is a Pythonbased free and open-source web framework that follows the model-view-controller (MVC) architectural pattern. Django provides some application which can build a website such as authentication system, administrative interface, a framework for creating GIS application. Django also has a smooth learning curve and significant communities. Google Cloud Platform (GCP) is a suite of computing services hosted by Google. There are three components used in this project: Compute Engine, Cloud Storage, and Cloud SQL. The Compute Engine hosts the Django server and Python script. The Cloud Storage stores the binary file and the Cloud SQL hosts the MySQL server to store the metadata of the datasets and the Django backend data. Figure 3-1 describes the architecture design for this project. 


\section{SNARC Data Portal Porject \\ Infrastructure Design}

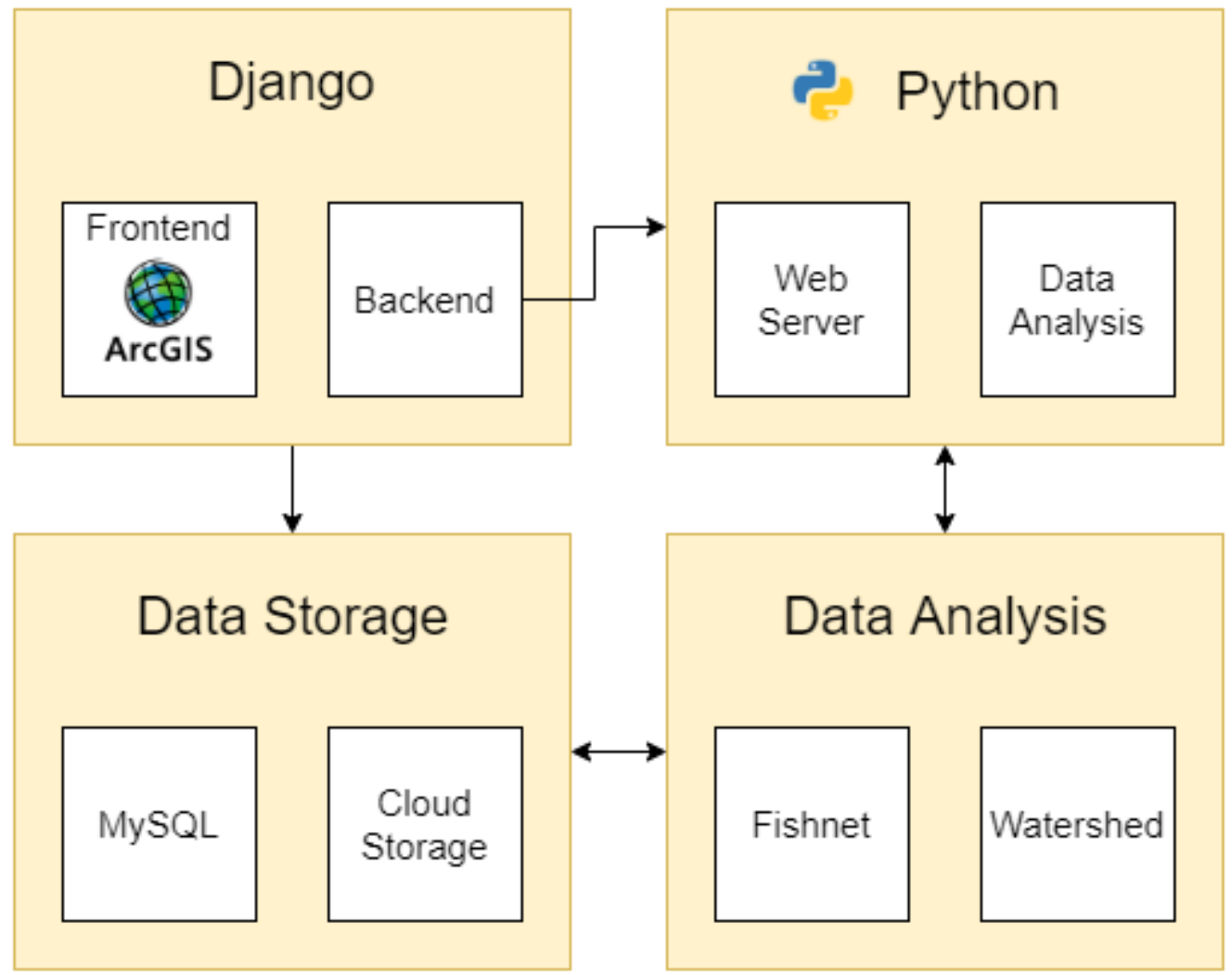

Figure 3-1: System Design

The ArcGIS API has been widely used in the geological analysis tool, such as calculating the intersect of two areas. The ArcGIS Online was used as a pipeline tool that can transfer the local data from ArcGIS Online to the web portal with ArcGIS online API. The ArcGIS Online also can display the web map in the web portal.

The ArcGIS was widely as a processing tool in this project. All the fishnet and study area feature layers were generated by ArcGIS Pro. The ArcGIS server is necessary if the ArcGIS Pro or Python SDK runs on the server. Considering the budget of this project, the intersect tool was rewritten with Python to avoid using in ArcGIS Pro when the web portal is up running.

\subsection{Project Plan}

A plan was developed at the start of the project to measure progress. The project has been divided into five stages: planning, design, development, test, and deploy. Table 2 explains the detail of the original project plan.

Table 3-2: Project Plan 


\begin{tabular}{|c|c|c|c|c|c|c|}
\hline & (1) & Name & Work & Duration & Start & Finish \\
\hline 1 & 숀 & ĐProject & 223.984 ho... & 132.49 day... & $12 / 20 / 198: 00 \mathrm{AM}$ & 6/23/20 11:55 AM \\
\hline 2 & & ĐPlan & 40 hours & 25 days? & $12 / 20 / 198: 00 \mathrm{AM}$ & 1/23/20 5:00 PM \\
\hline 3 & & Requirements collect & 16 hours & 10 days? & $12 / 20 / 198: 00 \mathrm{AM}$ & $1 / 2 / 20$ 5:00 PM \\
\hline 4 & & data collect & 8 hours & 5 days & $1 / 3 / 208: 00 \mathrm{AM}$ & 1/9/20 5:00 PM \\
\hline 5 & & Scope & 8 hours & 5 days? & 1/10/20 8:00 AM & 1/16/20 5:00 PM \\
\hline 6 & & Project Plan Documentation & 8 hours & 5 days? & 1/17/20 8:00 AM & $1 / 23 / 20$ 5:00 PM \\
\hline 7 & & ĐDesign & 23.984 hou... & 14.99 days? & 1/24/20 8:00 AM & 2/13/20 4:55 PM \\
\hline 8 & & UI Design & 8 hours & 5 days? & $1 / 24 / 208: 00 \mathrm{AM}$ & $1 / 30 / 20$ 5:00 PM \\
\hline 9 & & Frontend design & 7.984 hours & 4.99 days? & 1/31/20 8:00 AM & $2 / 6 / 204: 55$ PM \\
\hline 10 & & Backend design & 8 hours & 5 days? & $2 / 6 / 204: 55$ PM & 2/13/20 4:55 PM \\
\hline 11 & & Infastructure Design & O hours & 0 days? & $2 / 13 / 204: 55 \mathrm{PM}$ & $2 / 13 / 204: 55$ PM \\
\hline 12 & & ĐDevelop & 88 hours & 55 days? & $2 / 13 / 20$ 4:55 PM & 4/30/20 4:55 PM \\
\hline 13 & & Frontend develop & 32 hours & 20 days? & $2 / 13 / 204: 55 \mathrm{PM}$ & $3 / 12 / 204: 55$ PM \\
\hline 14 & 菭 & Backend develop & 32 hours & 20 days? & $3 / 12 / 204: 55$ PM & $4 / 9 / 204: 55$ PM \\
\hline 15 & & Database develop & 8 hours & 5 days? & 4/9/20 4:55 PM & 4/16/20 4:55 PM \\
\hline 16 & & Infastructure develop & 16 hours & 10 days? & 4/16/20 4:55 PM & 4/30/20 4:55 PM \\
\hline 17 & & 曰Test & 36 hours & 20 days? & 4/30/20 4:55 PM & 5/28/20 4:55 PM \\
\hline 18 & 若 & Design test plan & 16 hours & 10 days? & $4 / 30 / 204: 55$ PM & $5 / 14 / 204: 55$ PM \\
\hline 19 & & Develop test script and tes 1 & 8 hours & 5 days? & $5 / 14 / 204: 55 \mathrm{PM}$ & $5 / 21 / 204: 55 \mathrm{PM}$ \\
\hline 20 & & Report and fix bug & 8 hours & 5 days? & $5 / 21 / 204: 55 \mathrm{PM}$ & $5 / 28 / 204: 55 \mathrm{PM}$ \\
\hline 21 & & Docuentation review & 4 hours & 2.5 days? & $5 / 21 / 204: 55 \mathrm{PM}$ & 5/26/20 11:55 AM \\
\hline 22 & & ĐDeploy & 28 hours & 17.5 days? & $5 / 28 / 204: 55$ PM & $6 / 23 / 2011: 55 \mathrm{AM}$ \\
\hline 23 & & Deploy & 8 hours & 5 days? & 5/28/20 4:55 PM & $6 / 4 / 204: 55$ PM \\
\hline 24 & & Live test & 4 hours & 2.5 days? & $6 / 4 / 204: 55$ PM & $6 / 9 / 2011: 55$ AM \\
\hline 25 & & Finnal report & 16 hours & 10 days? & $6 / 9 / 2011: 55$ AM & $6 / 23 / 2011: 55$ AM \\
\hline
\end{tabular}

The development and test part takes the longest time in this plan. This period can be divided into three different phases. The first one is to build a basic demo of the web portal. Based on the review from the client to plan the next step of the project. The second phase is to finish the main feature of the data portal and deploy it. The last phase is the live test, technical documentation review, and writing the final report.

The original plan was scheduled based on the requirements and the deadline. The project followed the plan until the first demo. After the first demo, more comments and data samples were sent by the client, and those data were stored in several different formats that the system could not automatically analyze them. To solve this problem, a new design of the metadata collecting system replaced the automatic analyze system. It took some extra time to redo this part, but the schedule did not change too much because the original plan had some buffer time.

\subsection{Summary}

This chapter addressed the detail of the web portal design and the method of ArcGIS API used in this project. The project plan changed because of the client's new requirements. These changes were necessary and meet the requirements of the clients better. Chapter Four will include the database design in this project. 



\section{Chapter 4 - Database Design}

Chapter Four explains the data model and database design for the project. Section 4.1 describes the conceptual data model. Section 4.2 introduced the logical database model and fields of each individual table. Section 4.3 included the logical data model, and the last Section 4.4 is the summary of this chapter.

\subsection{Conceptual Data Model}

The conceptual data model displays the associations between the classes in the Django web platform and geological analysis tools. Figure 4-1 shows the method to store the user data and analysis tool data.

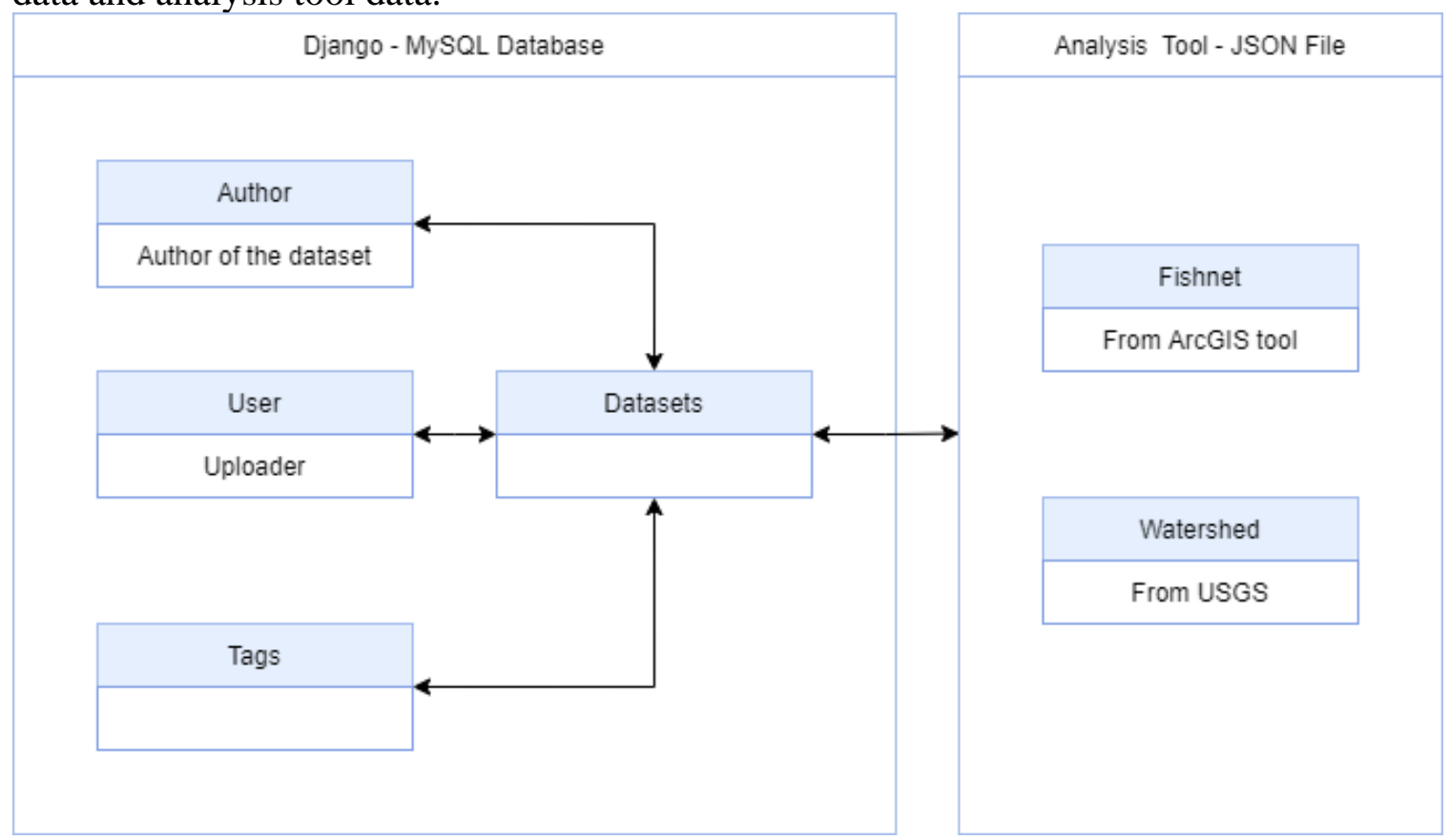

Figure 4-1: Conceptual Data Model

When the user uploads a new dataset, the system will store the uploader's id for permission control. All the metadata information collected from the data collection page will go through the analysis tools and find the fishnet and watershed of this dataset. The fishnet and watershed boundary data are stored in the JSON file format on the server. In this case, the frequency of that fishnet and watershed data change is low. The only operation used here is read operation. The JSON file can offer a better-read operation speed than a relational database. All the binary files such as CSV and shapefile will send to Cloud Storage.

The SNARC required a permission management system that different users can access the limited datasets base on the group. An extra table stores the group information in the Django backend. When the user sends a query request to the backend, the system will return the id of the dataset based on which group this user belongs to. 
All the datasets are presented as a number in the fishnet or a single point on the web map. So, the id of the fishnet which this dataset located in or the latitude and longitude should be stored in the datasets table. When the user sends a search request, the front end can generate a web map with the data detail on the fly and display it on the web portal with the ArcGIS API.

The attribute table in the ArcGIS web map synchronizes the data with the table in the MySQL database. When the user requests a fishnet or watershed view in the web map, it will display the ArcGIS Online web map directly.

\subsection{Logical Data Model}

The database of this project consists of two parts, which are the attribute table in the ArcGIS online feature layer, and the SQL database. The first part stores the total amount of datasets in each fishnet and watershed. The SQL database contained all the Django platform backend data and metadata for the datasets. Figure 4-2 shows the relationship between the SQL database and the attribute table.

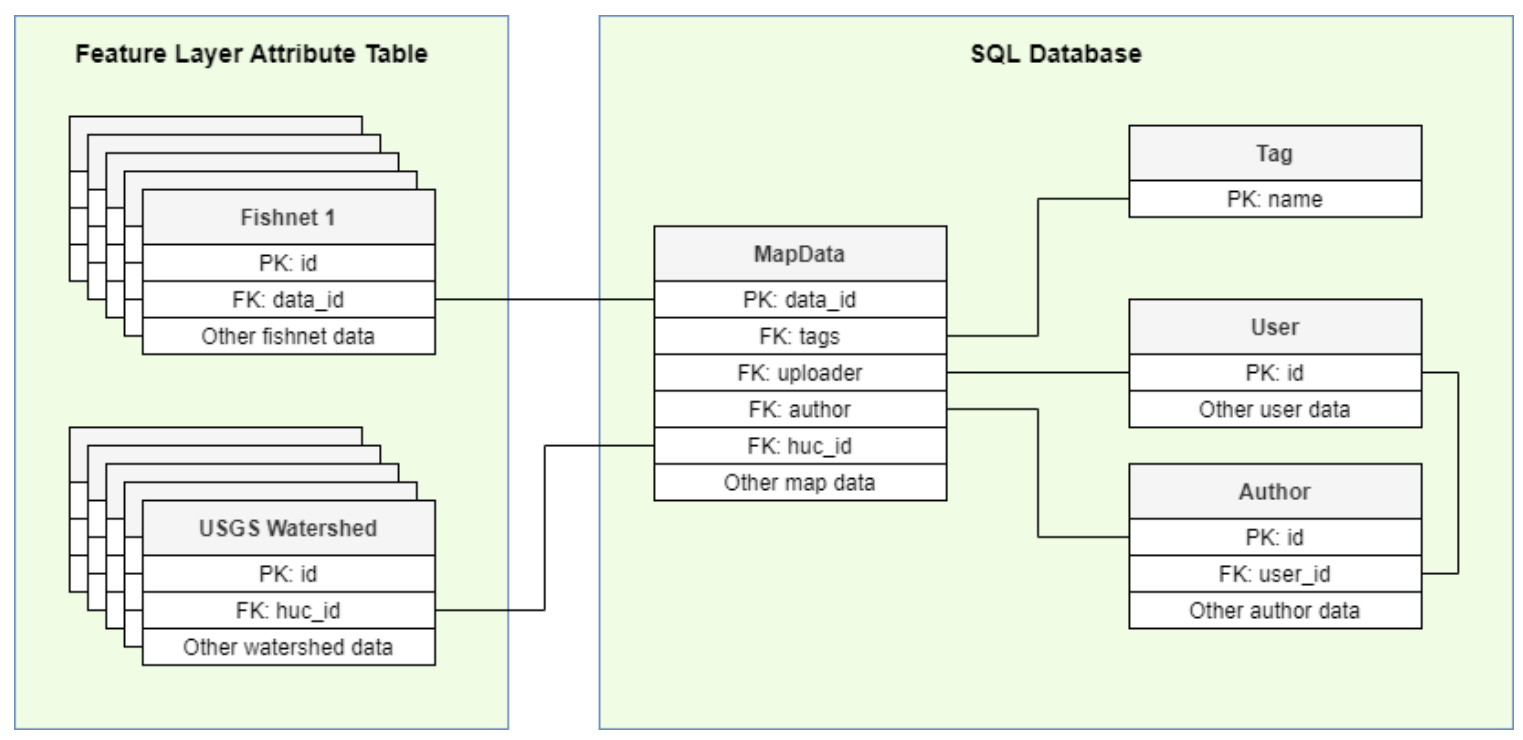

\section{Figure 4-2: Database design}

The fishnet feature layer contains the necessary information about the fishnet, such as id and boundary data. The field data_id stores the id of the datasets located in this fishnet. When the system receives the request, this web map can be displayed on the web portal. Different from the fishnet id. The watershed id only used for the search. The system built the connection between watershed and dataset with the watershed id. When a user sends a watershed id as a search request, the system can query only the datasets table to get the result.

The SQL database covers the metadata information of the dataset. The MapData table has four foreign keys connecting to the Tag, User, Author, and USGS watershed table. More detail of this table is in the SQL database design section. The user table contains all user's data in this data portal. The user recorded in the author table do not have to be a user in this organization. It includes all the essential information for an 
author. If the Author is a member of SNARC, the user can relate to the author with the foreign key in the author's table.

\subsection{Data Sources}

SNARC provided most of the data in this project. Researchers will upload the datasets to the data portal after deploy. The system was designed to collect the primary data information when the user uploads it. The fishnet and watershed data were calculated by the analysis tools. The fishnet boundary data were built with the fishnet geoprocessing tool in ArcGIS Pro based on the requirements from the client. The watershed boundary map was downloaded from the USGS official website.

The SNARC also provided some data like the California water flow map. Those data can be used in the research of the relation between the different water bodies.

\subsection{Summary}

The database design was essential for the success of this project. This project involved three different kinds of databases which are MySQL database for the Django and Python, Attribute table in a feature layer and JSON file for analysis tools. The next chapter is about how those data serve this project and the detail of the system implementation. 



\section{Chapter 5 - Implementation}

Chapter Five explains the implementation of the project. Section 5.2 introduces the system infrastructure design. The platform used to build the web application is Django and Python, so Section 5.3 is about the Django web server and Python script Section. 5.4 and 5.5 introduced the design of the fishnet analysis tool and watershed analysis tool.

\subsection{System Infrastructure Design}

The first step in the construction of the web application was building the development environment. The Google Cloud Platform host all the servers in this project. The web server and MySQL server are separated. The web server is serviced by the Compute Engine and MySQL database is serviced by Cloud SQL. For security considerations, the MySQL server is not open to the public. The connection between the web server and MySQL server protected by the IP address. The developing and production servers were deployed in two compute engines. The system of the production server is a clone from the developing server to ensure two servers have the same environment setting.

The operating system for the webserver is Ubuntu 18.04, using the Nginx as the reverse proxy. Nginx is open-source software for web serving, reverse proxy, load balancing, and more. However, Nginx cannot send the request directly to the Python application by itself. Python needs a hosting service to receive the HTTP request from the Nginx. The name of the hosting service is the Web Server Gateway Interface (WSGI). The UWSGI is often used for serving Python web applications. Figure 5-1 described how the Python application receives the request from the user. The end-user sent the request in the web browser to the server. The proxy server received the request and sent it to the web socket base on the configuration. The socket mapping with the UWSGI server and finally send the request to the Python web application.

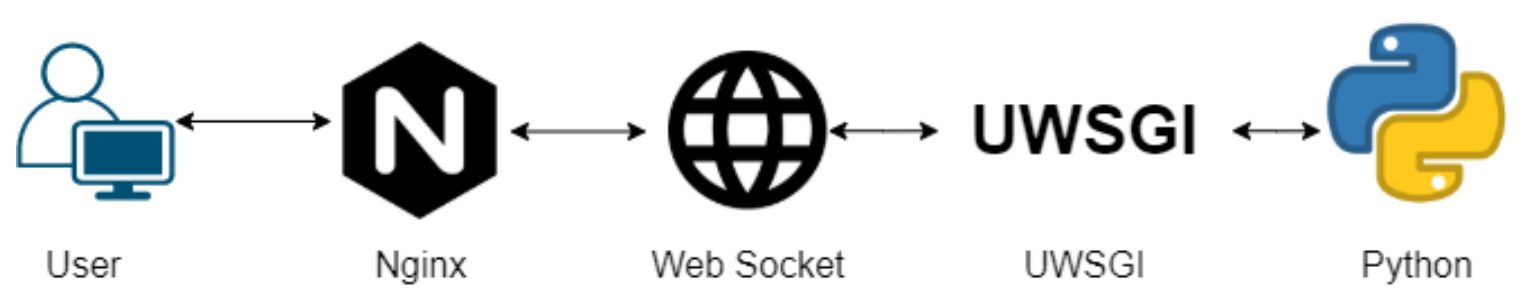

Figure 5-1: Technical Stack in the Linux server

The users can manage the dataset with the GUI frontend, which was built with Django and general web technology like HTML, CSS, and JavaScript. ArcGIS JS API generates the web map which is displayed on the webpage. The web server is also hosted by Django. The main functions of the Django webserver are routing, webpage render, and database connection. After the user uploads the dataset, some analysis tools will be triggered and retrieve the critical information from the dataset. Those scripts are built with Python. The data analysis tools include the fishnet tool and watershed tool. When 
the system receives a response from the analysis tools, all the data will be saved to the relational database, a MySQL database hosted on the GCP Cloud SQL service. The binary file will be sent to the Google Cloud Storage Service. Figure 5-2 shows the system architecture design.

Architecture: SNARC Data Portal Project

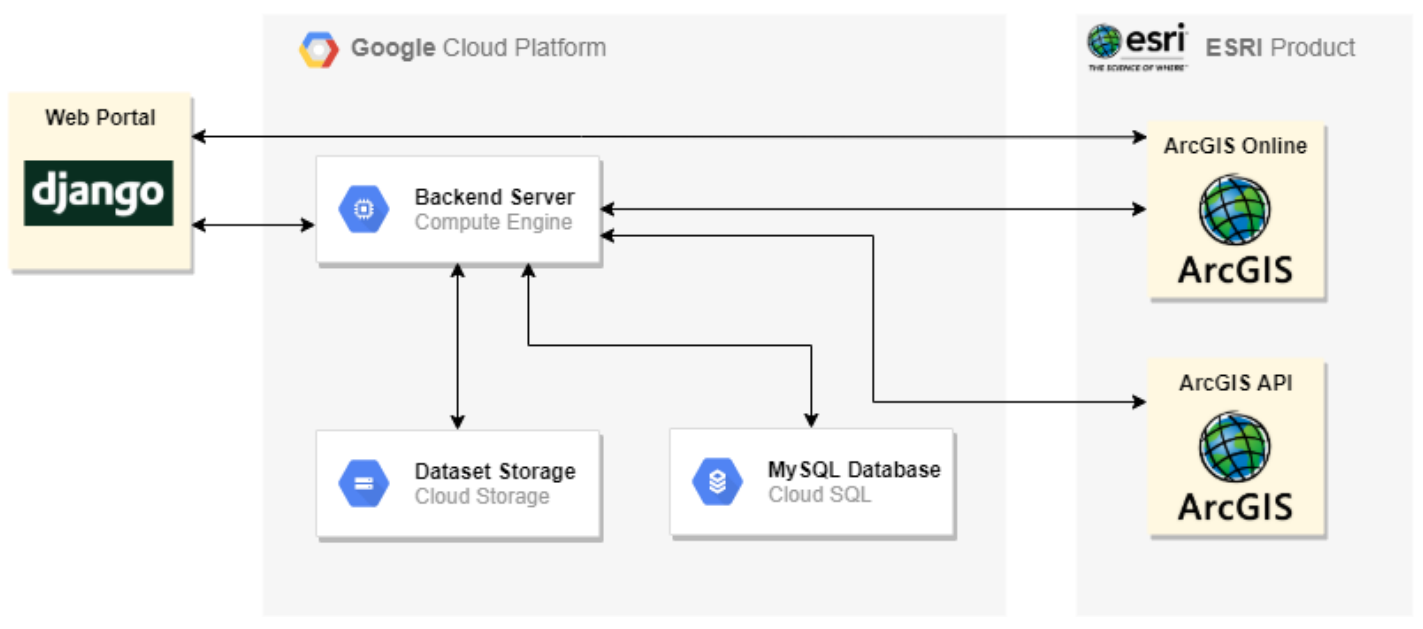

Figure 5-2: System Architecture Design

\subsection{Build a web application with Django}

There are four main functions that Django should provide in this project: allowing user to upload the data, displaying the data on the map, allowing the user to view the dataset detail and download, and managing the content and users with the admin page in this data portal.

When a user uploads the new dataset to the data portal, the system should collect some metadata about this dataset. For a GIS dataset, the location of the data collected is indispensable. The first step is to get the geolocation about this dataset. The system gets the location with ArcGIS sketch widget API. This API can create a widget in the web map that allows the user to draw a point, line, and polygon on the map and receive the location information with the API. In this case, the user can click draw a pointed tool and drop a point on the map. The web map can be zoomed in or out to adjust accuracy. Figure 5-2 shows the interface to collect location data. When the user picks a location and clicks the next button, the latitude and longitude data is stored in the user session for later use. 
Please use 'Draw a Point' tool on the upper right corner of the map to select a location of dataset.
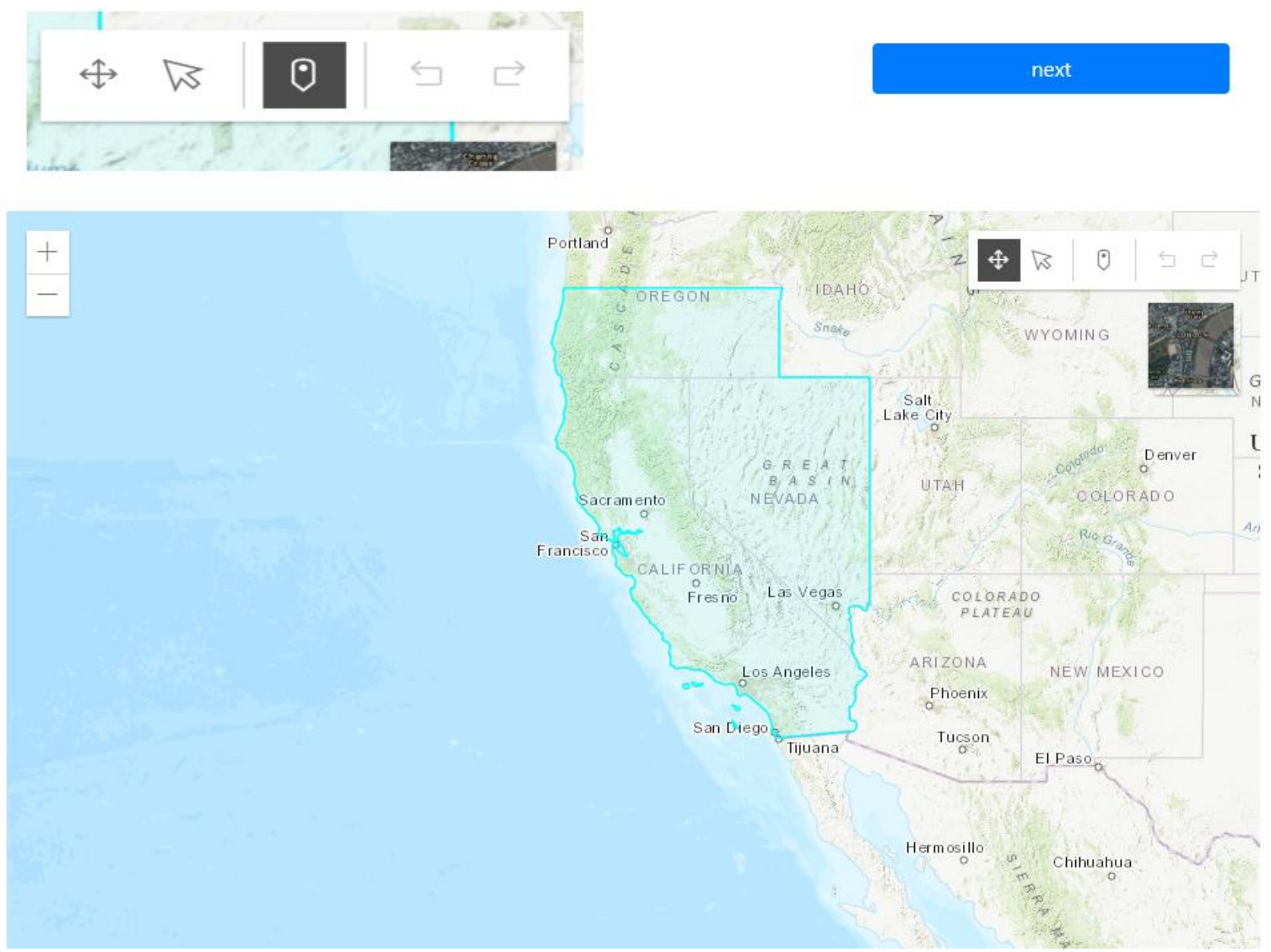

Figure 5-3: Location Picker

The next step of dataset upload is to collect the metadata. The template of metadata is from USGS. Figure 5-4 shows the UI of the metadata collection page. This page built with the HTML form. The HTML form is a fundamental element to collect user input. The form on this page can collect the user input and check the data validation. When the user clicks the submit button, the system will send the form to the backend and trigger more functions such as file upload and data analysis tools. The next step is to design a page that can display that information. 


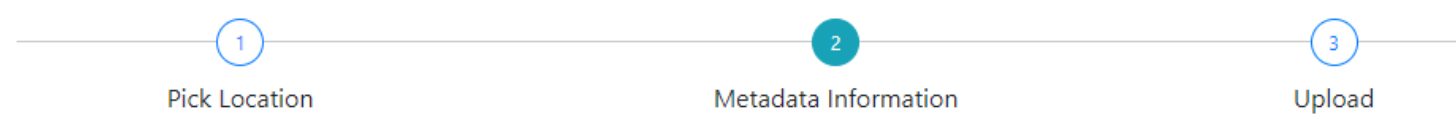

\section{Metadata Collection}

Title:

Title

Abstract:

Abstract

Begin date:

YYYY-MM-DD

End date:

YYYY-MM-DD

Author:

Author1

\section{Figure 5-4: Metadata collection page}

The dataset display page consists these parts: a web map, a list of datasets, and a search box. The web can display the existing datasets in two different views. The first one is shown in Figure 5-5. The label on the fishnet square shows the number of datasets in this area. The map shown here is a web map hosted on the ArcGIS Online. When the user chooses this view, the system will call the ArcGIS JavaScript to display the web map here. The dataset id is stored in the feature attribute table. There is a total of five feature layers to cover all the scales. Another view is to show the dataset location as a point on the map, this method used on-fly map when the system receives the request from the browser, one scrip in the backend will get the current map extent information and return the dataset id located in this area. The list on the left side can be dynamically changed with the map zoomed in or out. 


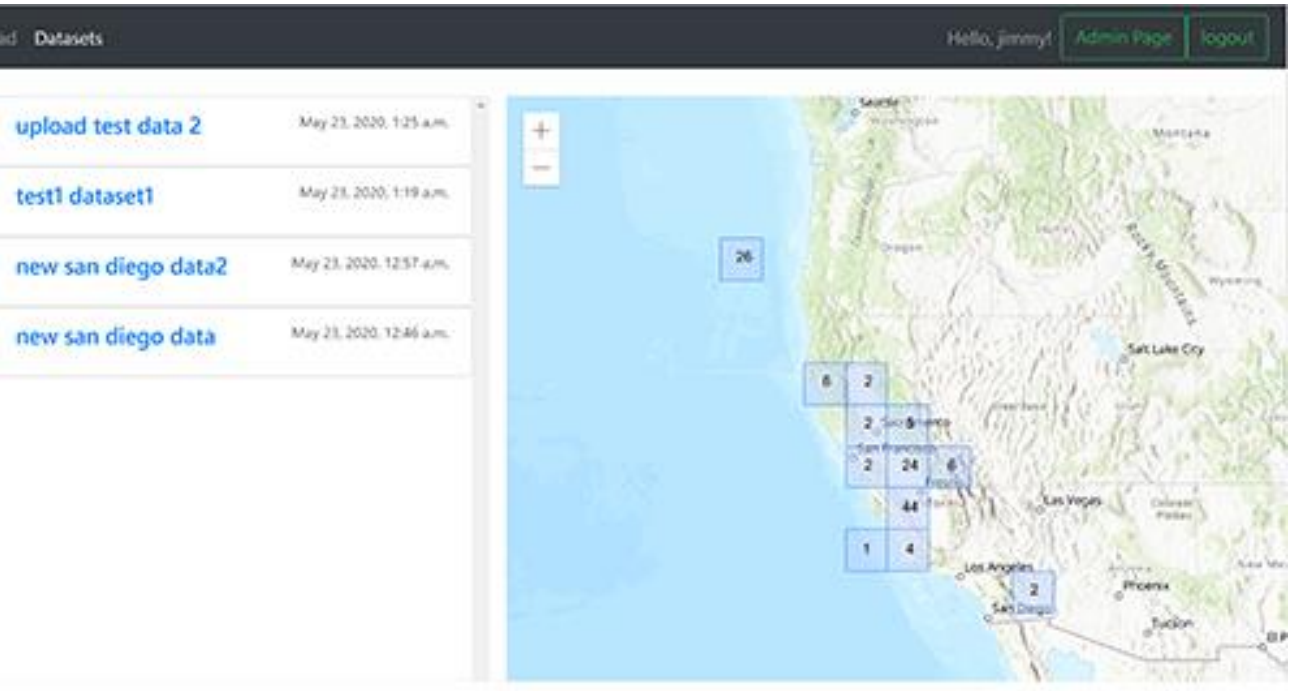

\section{Figure 5-5: Dataset Display Page}

By clicking the title of the dataset, a user will be redirected to the dataset detail page. As Figure 5-6 shows, this page can display all the detailed information of this dataset and allows users to download the dataset. This page directly queries all the information from the MySQL database and displays them here. In the Django configuration, all the binary data in this project are store in cloud storage. When the user click the download button, the file will sent directly from the Google cloud storage to the user's disk. 


\title{
Dataset Detail
}

\author{
description.
}

\section{new san diego data}

Abstract

orem ipsum dolor sit amet, consectetur adipiscing elit. Aliquam aliquet consequat erat, et feugiat magna sodales at. Cras et purus tempor, commodo ante ut, maximus urna. Pellentesque posuere accumsan vulputate. Maecenas tortor quam, accumsan id laoreet sit amet, dapibus vitae nulla. Aliquam pulvinar pellentesque dolor eu fringilla. Curabitur at ex convallis, rutrum augue ac, varius libero. Nullam imperdiet purus ac ex elementum, quis euismod sapien condimentum. Maecenas dignissim vel libero vitae viverra. Aenean sit amet tempus dui, et eleifend arcu. Donec rhoncus aliquet nunc. Integer suscipit purus sed imperdiet mattis. Suspendisse potenti. Sed ut nunc et massa dignissim malesuada vitae sit amet massa. Sed ac magna in purus congue gravida nec vel lectus. Cras pharetra porta arcu ut feugiat. Nullam rhoncus tincidunt leo, scelerisque facilisis risus bibendum vel. Suspendisse turpis tellus, euismod ac erat vitae, iaculis suscipit nibh. Aenean porttitor diam neque, nec consequat erat gravida a. Aenean sodales tincidunt elit, in iaculis ante volutpat sed. Nullam purus orci, lobortis eu euismod eget, bibendum eu velit. Maecenas suscipit pulvinar arcu, a imperdiet sem gravida sit amet. Vestibulum blandit velit pretium ipsum finibus mattis. Fusce pharetra dolor eget turpis ultricies tristique. Suspendisse mattis, purus vel molestie tincidunt, nisl massa ullamcorper leo, sed tincidunt ipsum eros at dolor. Suspendisse potenti. Duis feugiat odio at lacus mattis iaculis. Duis placerat diam magna, id venenatis neque molestie sit amet. Aliquam ut efficitur tellus. Proin mattis ultrices lacus, id ullamcorper sem convallis at. Phasellus quis hendrerit magna, sed imperdiet quam. Nunc lacinia purus vel enim fringilla pharetra.

\section{Figure 5-6: Dataset Detail Page}

\section{Key Information}

Download this dataset

Download

Tags

tag3

Author

Author1

HUC Information

181002041505

Start Date

Nov. 11, 2011

End Date

Nov. 11, 2020

Uploader

jimmy

IInlnar data

The last primary function of Django is the admin page. Django provided an admin system that a superuser can manage the content, users, and permissions. Figure 5-7 is the design of the admin page.

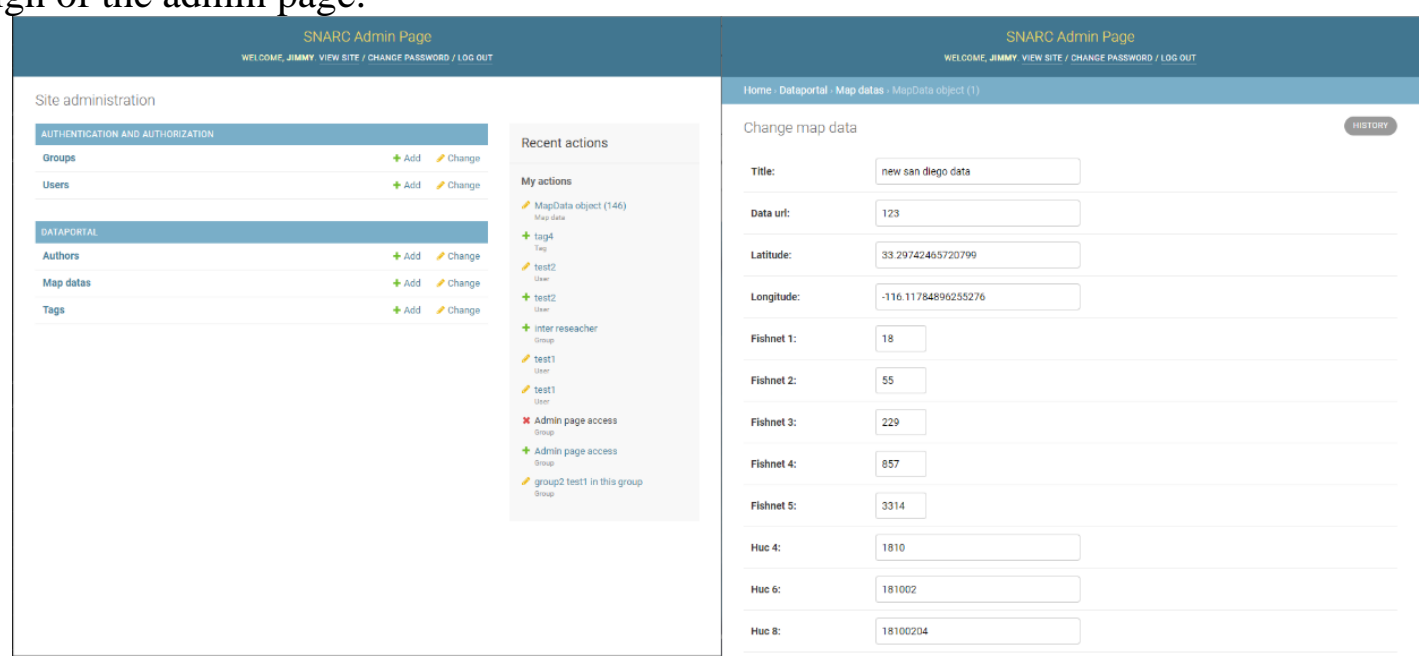




\section{Figure 5-7: Admin Page}

The user register system has been banned in this system. Only the superuser can create new users. The superuser can manage all the content and users in this portal, and a regular user can just manage the content they uploaded. The default settings of the system have been rewritten to limit the permission. All users at least belong to one group. A regular user only can access the content uploaded by the user in the same group. When the user requests the data in the front, the system will check the groups this user belongs to and query the data based on the group information.

\subsection{Fishnet analysis tool}

The fishnet tool is designed to display the dataset location intuitively. The original fishnet used in this project is generated by the create fishnet geo-processing tool in ArcGIS Pro. As Figure 5-8 shows, the parameters are the extent cover the study area, and the number of row and column should be 10 . It will generate a fishnet contain 100 square areas in it. This fishnet is marked as fishnet_1.

\begin{tabular}{l|l|l|r|}
\hline Geoprocessing & & \\
\hline & & \\
\hline
\end{tabular}

Figure 5-8: Fishnet Tool

Each fishnet area has an id, increasing from the bottom left to top right. Figure 5-9 is the fishnet with ids. A new field was added to the attribute table to store the id of the datasets located in it. Double the row and column number and repeat the fishnet creation four times. The results of this are five fishnets with 10x10, 20x20, 40x40, 80x80 and 160x160 marked as fishnet_1 to fishnet_5. Those layers can cover all visibility when the user changed the scale in the web map. Each square area in fishnet_1 to fishnet_4 contained four smaller square in it. So, the next step is to find the relation between 
different fishnets.

\begin{tabular}{|c|c|c|c|c|c|c|c|c|c|}
\hline \multirow[b]{2}{*}{91} & \multirow[b]{2}{*}{92} & \multirow[b]{2}{*}{93} & \multicolumn{2}{|c|}{ Oregon } & \multirow[b]{2}{*}{96} & \multirow[b]{2}{*}{97} & \multirow[b]{2}{*}{98} & \multirow[b]{2}{*}{99} & \multirow[b]{2}{*}{100} \\
\hline & & & 94 & 95 & & & & & \\
\hline 81 & 82 & 83 & 84 & 85 & 86 & 87 & 88 & 89 & 90 \\
\hline 71 & 72 & 73 & 74 & 75 & 76 & 77 & 78 & 79 & 80 \\
\hline 61 & 62 & 63 & 64 & 65 & 66 & 67 & 68 & 69 & 70 \\
\hline 51 & 52 & 53 & 54 & 55 & 56 & 57 & 58 & 59 & 60 \\
\hline 41 & 42 & 43 & 44 & $\begin{array}{r}45 \\
\text { Cal }\end{array}$ & & 47 & 48 & 49 & 50 \\
\hline 31 & 32 & 33 & 34 & 35 & 36 & 37 & 38 & 39 & 40 \\
\hline 21 & 22 & 23 & 24 & 25 & $26^{\operatorname{Los}}$ & $\ln _{27} 7^{5}$ & 28 & 29 & $3 \theta_{\text {hoen }}$ \\
\hline 11 & 12 & 13 & 14 & 15 & 16 & .17 & 18 & 19 & 20 \\
\hline 1 & 2 & 3 & 4 & 5 & 6 & 7 & 8 & 9 & 10 \\
\hline
\end{tabular}

Figure 5-9: Fishnet_1 for build the fishnet tool

Figure 5-10 shows the relationship between the fishnet_1 and fishnet_2 in the bottom left corner. The method to build the relationship between different fishnet is to bring the fishnet into a matrix to calculate with the row and column number. The row and column number connection between two fishnets are:

$$
n_{1}=\operatorname{round}\left(\frac{n_{2}}{2}\right)
$$

The $\mathrm{n} 1$ means the row or column number in smaller fishnet, and $\mathrm{n} 2$ means the larger one. With the new row and column number, the id_1 can be calculated with this equation:

$$
i d_{1}=\left(\left(\text { length }_{\text {fishnet }}-\text { row }_{1}-1\right) * \text { lengt }_{\text {fishnet }}\right)+\text { column }_{1}+1
$$

The length $h_{\text {fishnet }}$ is the length of the fishnet, the length of the fishnet_1 is 10 and fishnet_2 is 20. If the fishnet id of a dataset in fishnet_2 is 108, the method to calculate the id in fishnet_1 is:

1. Find the row and column of 108 , row $=14$, column $=7$

2. Calculate the row and column number in fishnet_1 round $\left(\frac{14}{2}\right)=7$ and round $\left(\frac{7}{2}\right)=3$, row $=7$, column $=3$

3. Get the id in fishnet_1 $((10-7-1) * 10+3+1=24$

With this method, the system can generate the id in all fishnet and update the attribute table when the new data uploaded. 


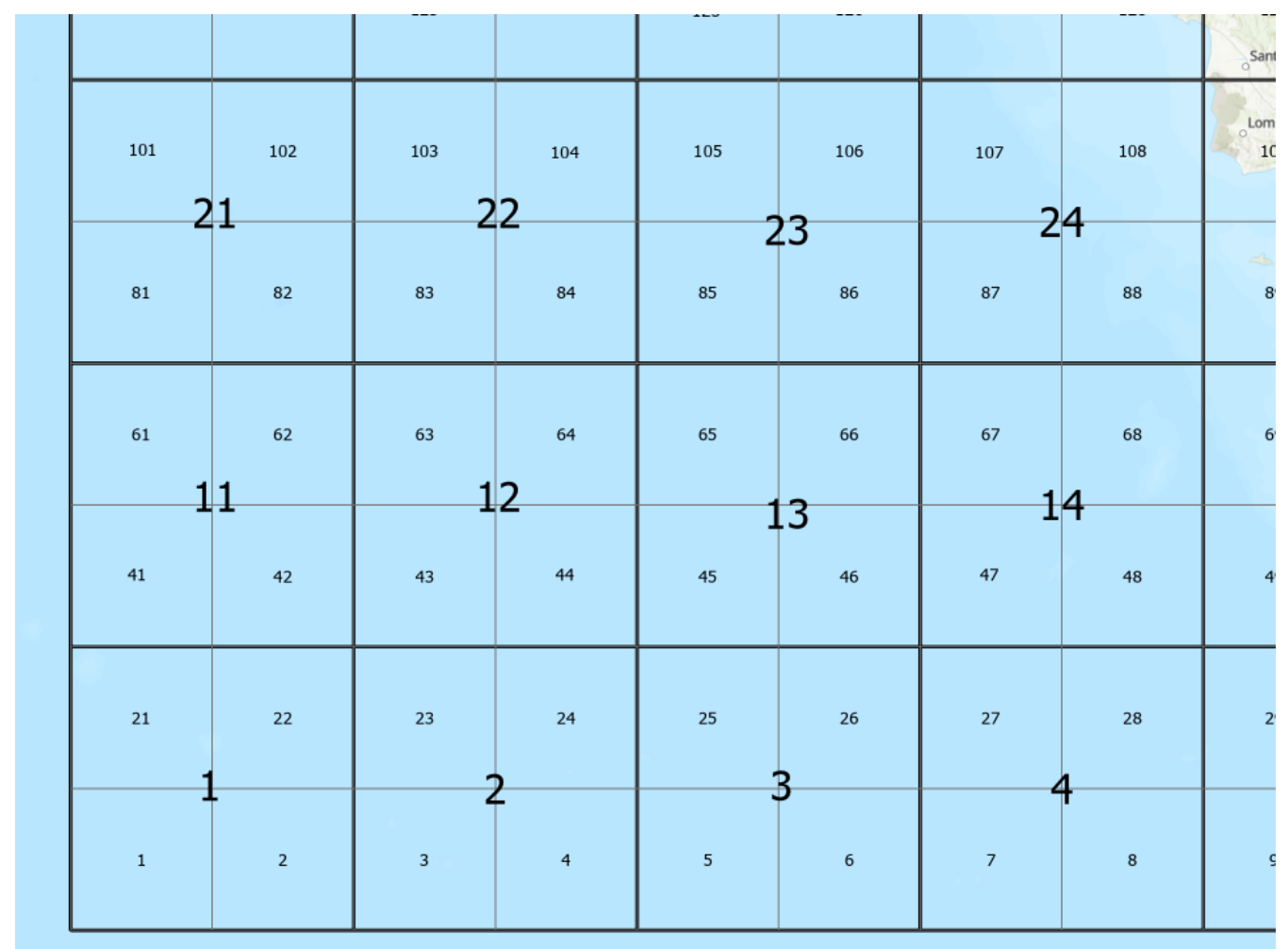

Figure 5-10: Relationship Between Fishnet_1 and Fishnet_2

\subsection{Watershed analysis tool}

One of the requirements from SNARC is tagging the dataset with the USGS watershed number. When the user searches the watershed number, the related datasets will be returned to the user.

The watershed layers used in this project were downloaded from the USGS official website. The watershed is used broadly in applications from scientific research to regulatory work. The watershed datasets contained six different layers, which are HUC-2, HUC-4, HUC-6, HUC-8, HUC-10, and HUC-12. Each area in HUC-2 covers a large area, and HUC-12 covers the smallest area. The individual area in HUC-2 covers the whole of California, so the HUC-2 layer did not include in this project. The number in the name of the layer also means the length of the watershed number. Every two digits connect the one area in each layer. For example, the watershed 1807 in HUC-4 covers the Los Angeles and San Diego areas. The area 18070106 in HUC-8, which just covers Los Angeles County, the area 1807 contains area 18070106, and the longer length of the id means the smaller area it covered. Figure 5-3 shows the watershed boundary HUC4 and the area 1804 in HUC8. The label is the id of each area. 


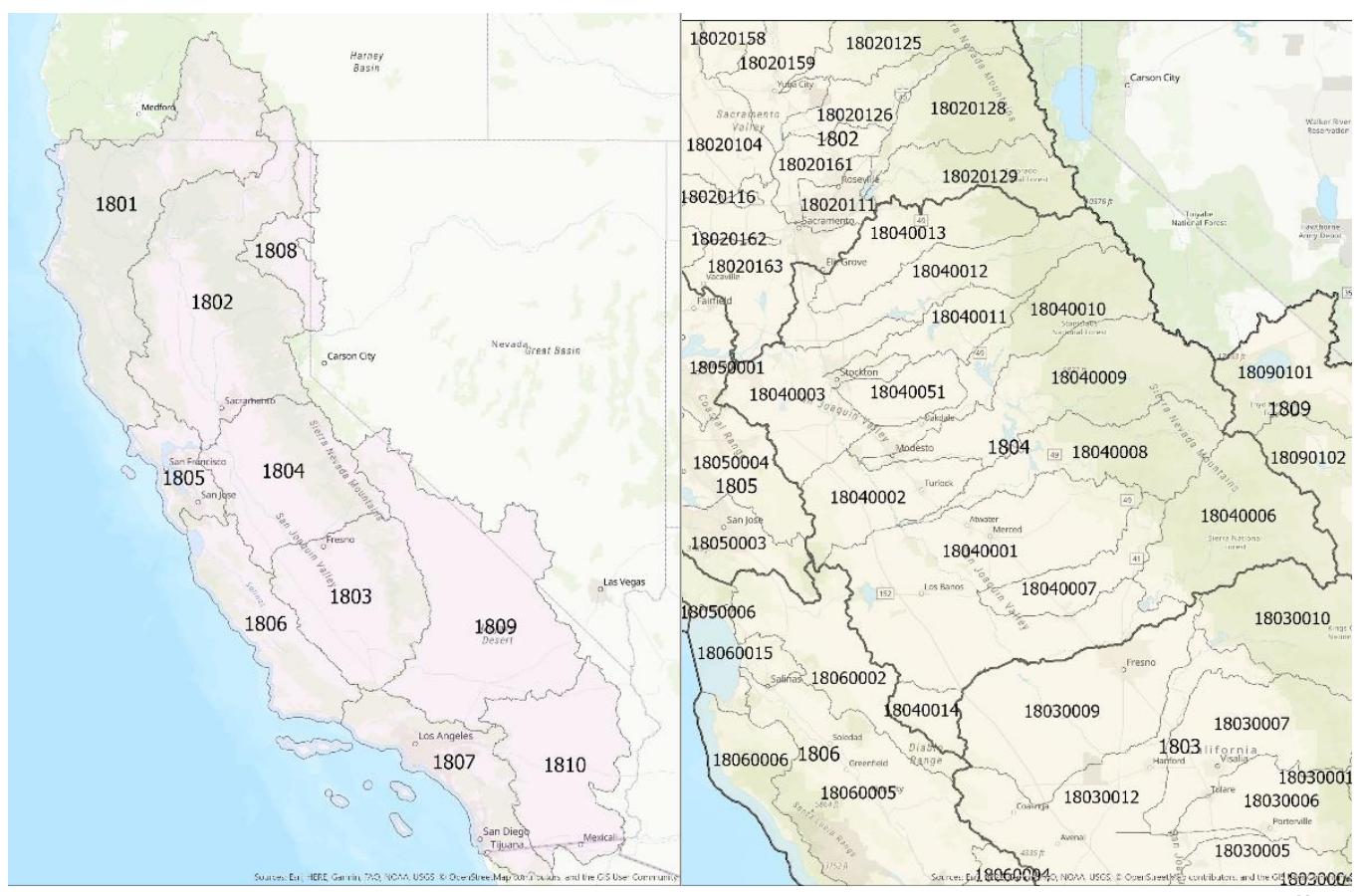

Figure 5-11: Watershed boundary with ID HUC4. Right: HUC8 zoom to the \#1804 area with ID

To find the relationship between the dataset and watershed, the Intersect in ArcGIS REST API was used to match the user uploaded dataset and watershed information. The intersect API receives the geometries data of two polygons and return the intersecting area of those two polygons. Considering the speed of the process, the first step is to save all polygon geometry information locally on the webserver. The local disk I/O is much faster than getting data from ArcGIS Online with the API. In this case, all watershed geometries data were saved in five JSON files. The reason for using the JSON file used here rather than the relational database is that the JSON file has faster reading speed. In this case, the frequency of the watershed boundary is low. Most of the action is read. When the watershed data is ready, the next step is implemented with the ArcGIS REST API.

Most of the area in the Sierra Nevada and watershed \#18 are intersected, but some area was not overlapped. Figure 5-12 shows the difference between the Sierra Nevada boundary and USGS watershed \#18 boundary. The area with red background is the Sierra Nevada boundary, and the black outline is the USGS watershed \#18. Scientists and researchers in SNARC, also mentioned some of their data are in the Nevada and Oregon. Use watershed \#18 as the study area is not enough for this project. 


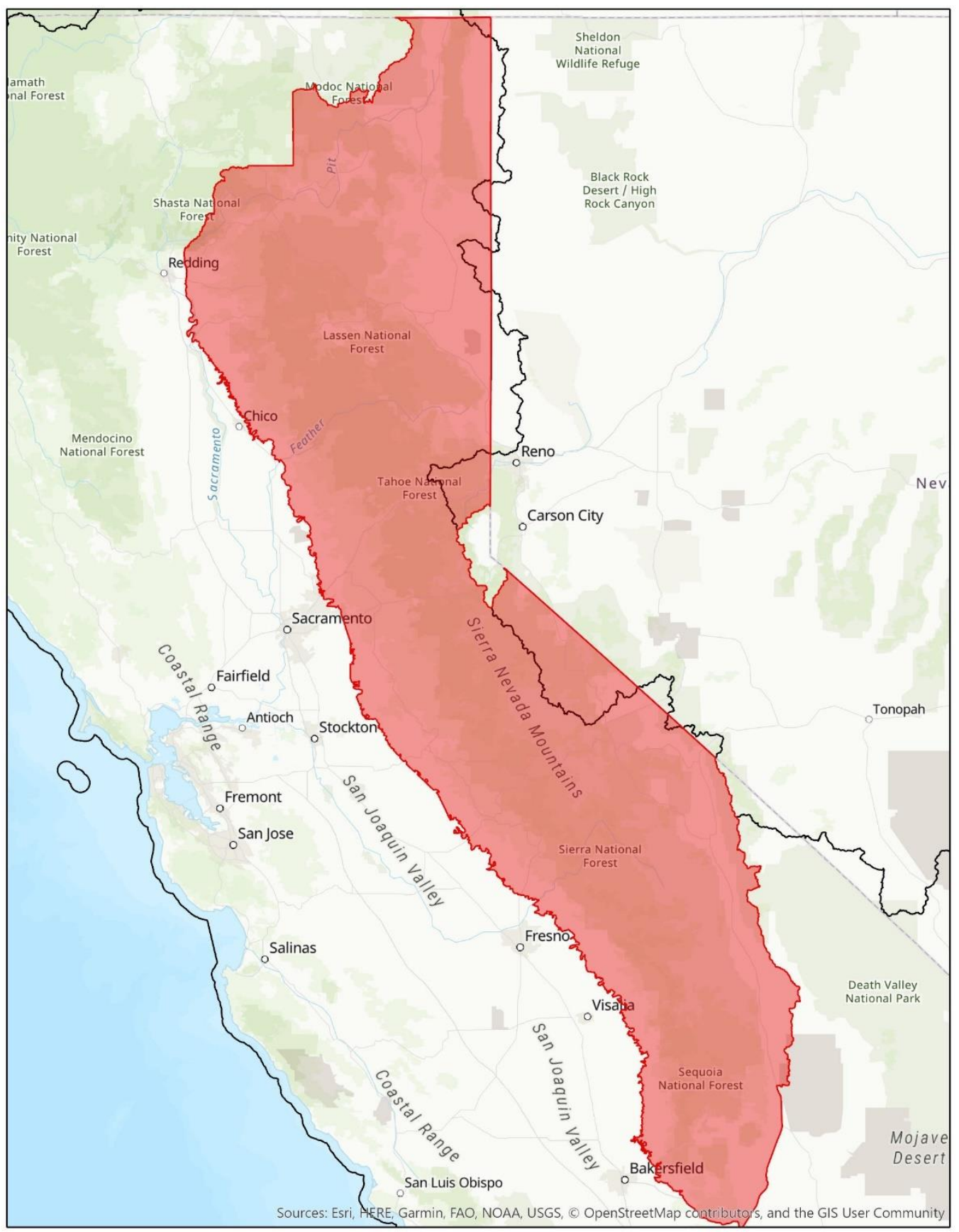

Figure 5-12: California State Boundary and USGS Watershed \#18 Boundary

Adding the watershed around the \#18 can solve this problem. Figure 5-13 displays the watershed around the Sierra Nevada Area. The boundaries with the black outline are watershed \#16, \#17, and \#18. However, the larger area means higher time complexity 
when the analysis tool is running. The solution is to use the state boundary, and the fishnet area limits the study. The blue background area is the fishnet boundary, and the red background is the state boundaries of California, Nevada, and Oregon.

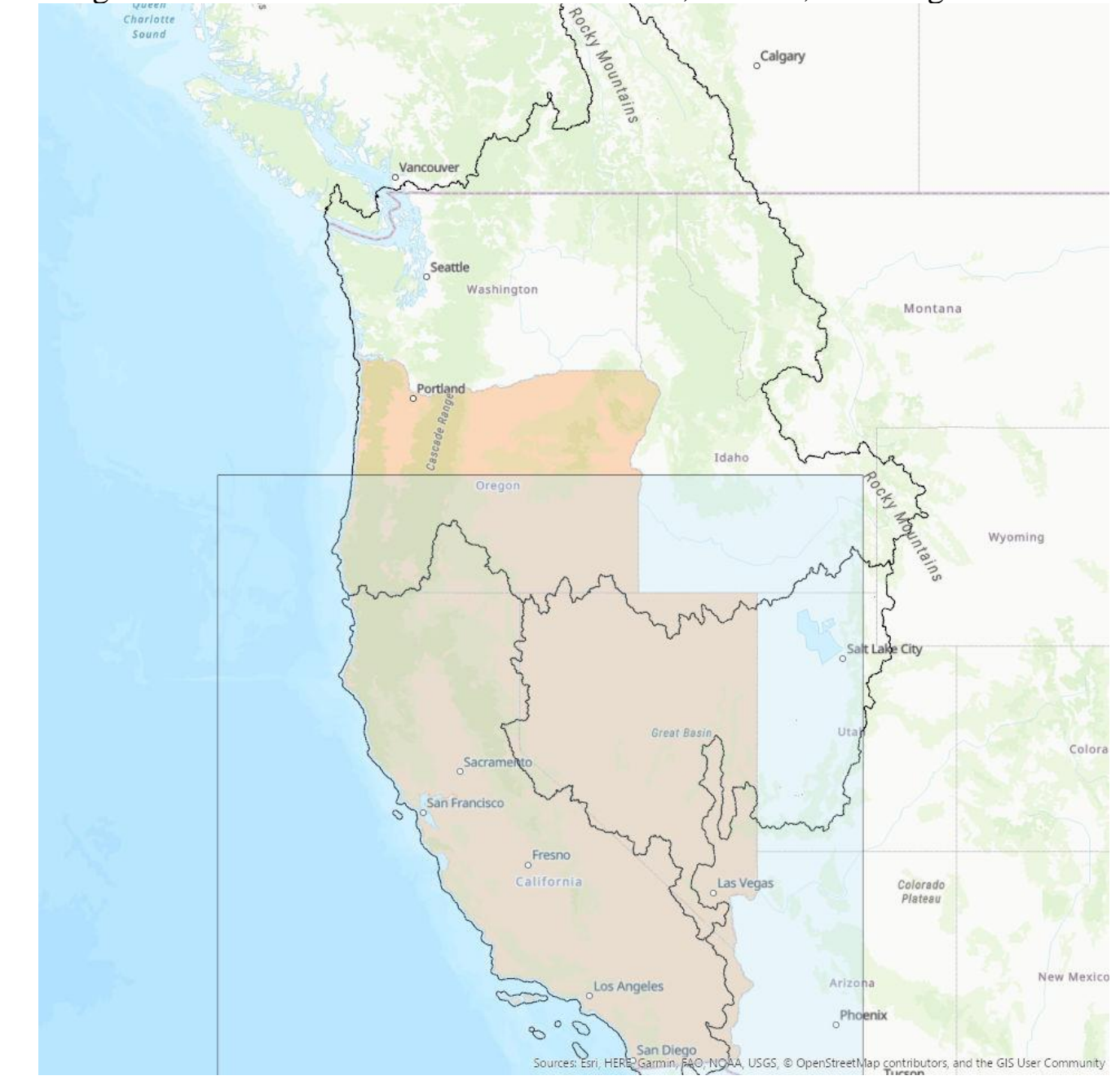

\section{Figure 5-13: The Fishnet, States, and Watershed Boundaries.}

The first step to generate the study area boundary is to merge the watershed areas. Use the merge tool in the ArcGIS Pro to merge the \#16, \#17, and \#18 in each HUC layers. The second step is to use the intersect tool to find the overlap area in different layers. The intersect can accept more than two layers as input. Use the boundary of three states, fishnet, and merged watershed boundaries as input, and the result is shown in Figure 5-14.

In this way, the attribute table of the intersect result includes all watershed areas detailed information, and the shape of the areas in the edge is modified by the intersect tool. 


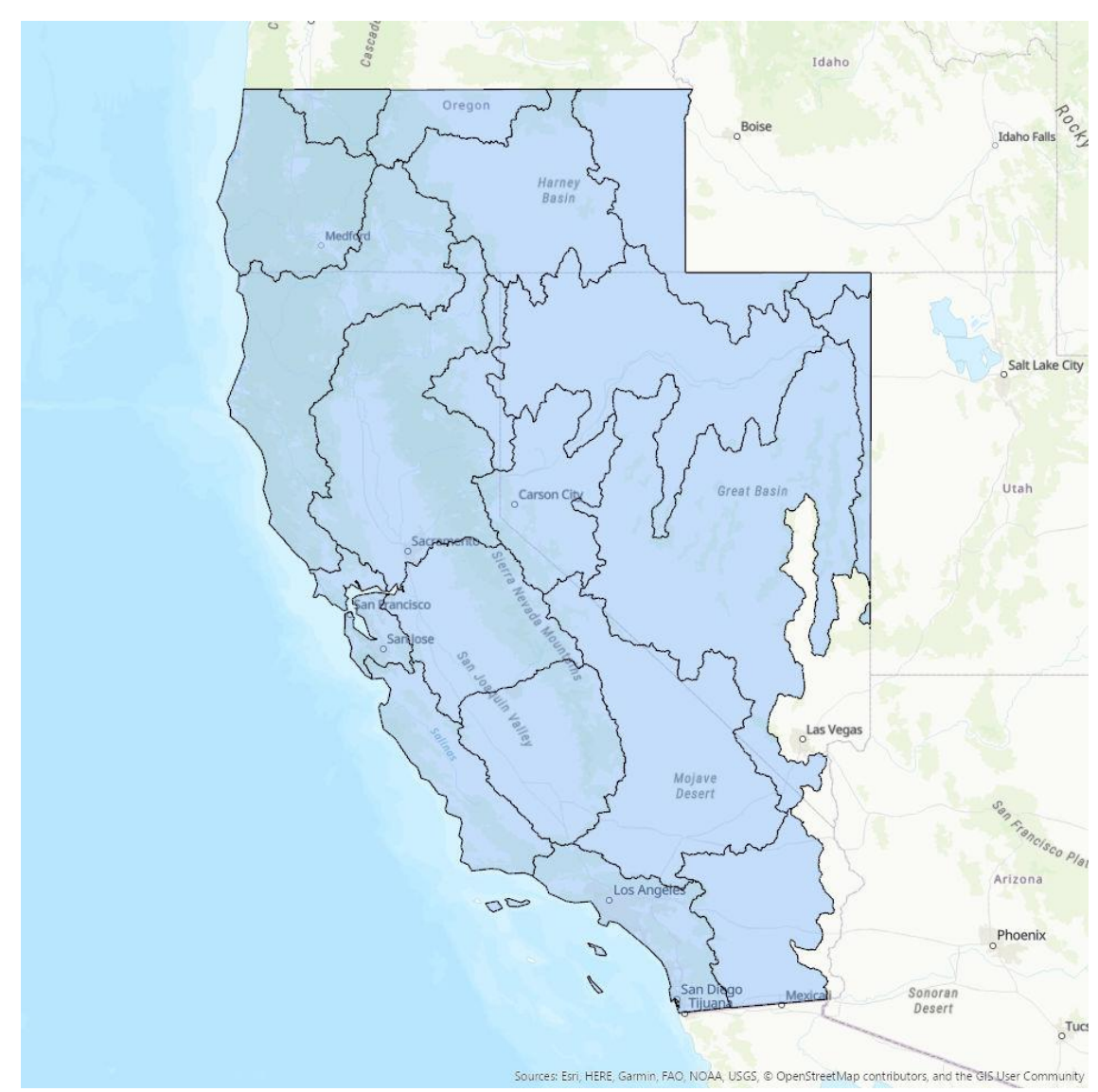

\section{Figure 5-14: Final Study Area with Watershed HUC-4 Boundary}

Base on the property of the watershed number, the HUC-12 number includes all other layer's information, but directly finding the HUC-12 number should compare each polygon in the HUC-12 layer with the user uploaded dataset location. There are more than 5000 polygons in HUC-12 just in the study area. The tool needed to go through all the 5000 polygons in the worst case. Due to the ArcGIS intersect API can only process 10 API calls per second. The entire process can take about ten minutes at most. The better way to find the number is to start from the HUC-4 layer and use the result to query in the next layer. Starting with the HUC-4 layer because it just has about 40 polygons in this layer. Using ArcGIS Interest API to compare each polygon with the uploaded dataset and find in which watershed this dataset is located. Finding the HUC-4 watershed number and using it to query the number starts with these four digits in HUC-6. Comparing the query result with the uploaded dataset can find the HUC-6 number. The searching is repeated to find the HUC-12 number. Because the number of the smaller polygons in each big polygon is no more than 40 , the maximum loop times are $40 * 5=200$. It is much less than 5000 loops otherwise searching directly from the HUC-12 layer. In this way, the system can find the watershed number in 10 seconds.

\subsection{Summary}

This chapter introduced the implementation of web application and analysis tools. Any dataset upload from the data portal will go through several analysis tools and store in the 
cloud storage. Figure 5-15 shows the user flow in this project. The next chapter will talk about the analysis result and the results of this project.

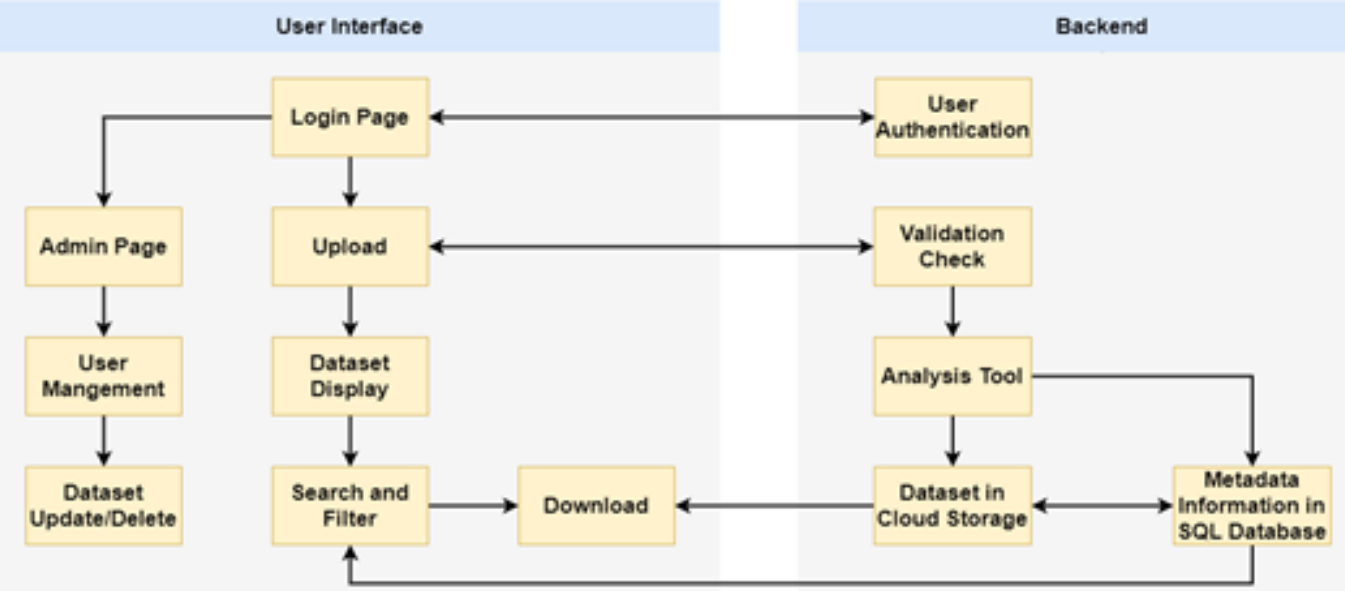

Figure 5-15: Project User Flow 


\section{Chapter 6 - Results and Analysis}

Chapter Six introduces the analysis and results of this project. When more data uploaded to this data portal, more analysis tools can be design to extract more information from the data. Section 6.1 introduces how the data can be reported and virtualize in this platform. Section 6.2 talks about what I get and the process for this project.

\subsection{Result and Analysis of the SNARC Datasets}

The data portal is not only the storage for data from SNARC but also a data analysis tool to help the SNARC know their data. This section will introduce how the analysis tools help the SNARC.

The fishnet analysis tool shows the geolocation information about the dataset. This information can help to evaluate the geolocation relationship between the datasets. For example, the fishnet information can visualize the data as a heat map to display the data volume in this area. The SNARC can plan future studies according to the heat map.

The watershed analysis tool presents the relation of the datasets based on the aquatic system. With the watershed information for each dataset, the researcher can focus on the relation of the watershed but not the individual dataset. When a new dataset is uploaded, the system can inspect the dataset to find which watershed this dataset belongs to. According to the relationship between a dataset and the watersheds, researchers can find the connection between each dataset. Compared with direct analyzing the dataset, this method avoids repetitive works and is more efficient.

As the number of dataset increase, the system can generate some reports based on the result of the analysis tool to guide the leader in SNARC to schedule future work. For example, with the dataset information, the system can find the geographical feature of the aquatic system and summarize the standard features. The data can also be used on the data visualization. According to some characters of the water body, the system can visualize the data on a map, displaying it with a different color. For example, Figure 6-1 shows the datasets count by the watershed. With this map, the researchers can get a big picture of the data distribution information. This map can also be displayed on the data portal. 


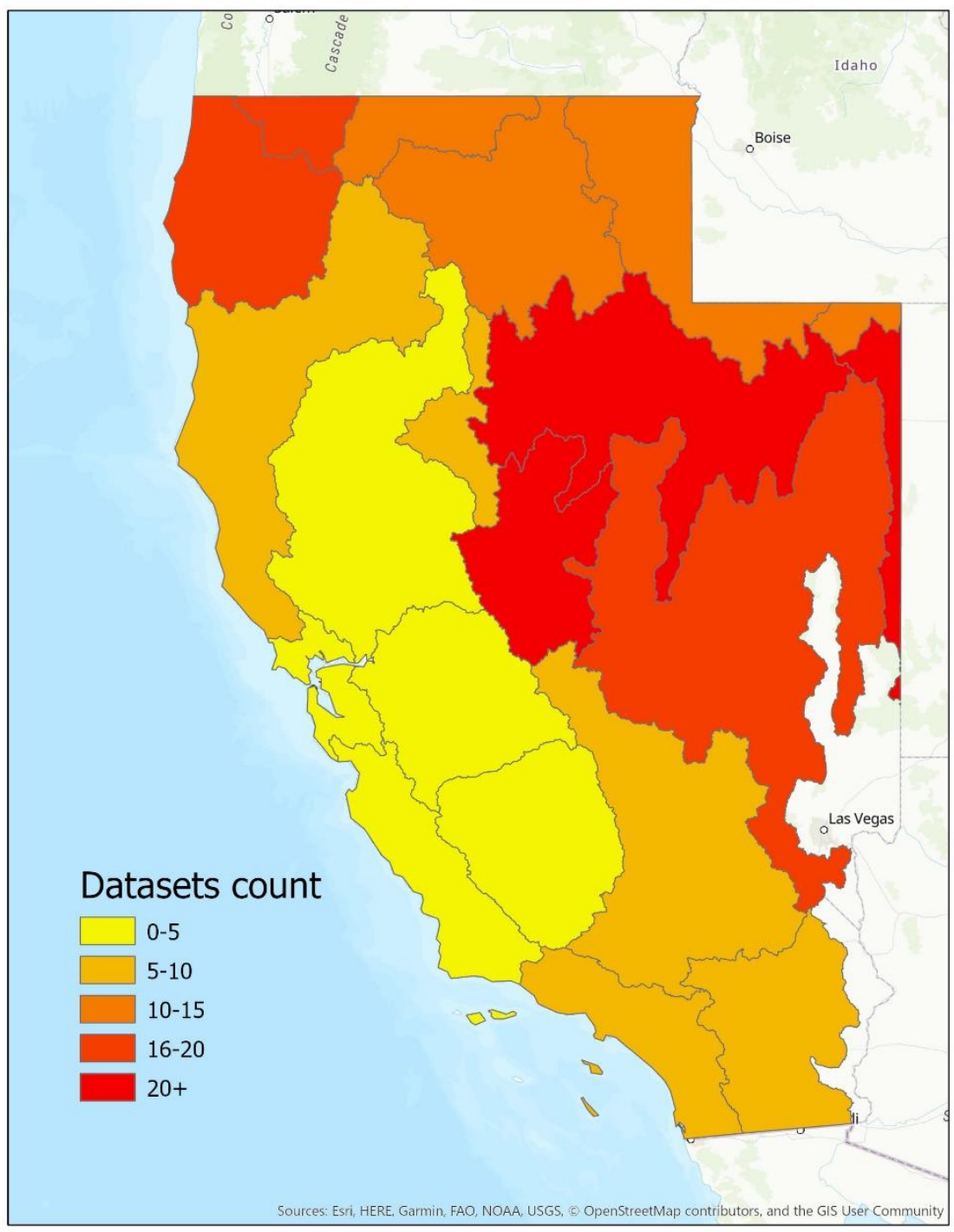

Figure 6-1: Datasets count by watershed

\subsection{Results}

The primary requirement from the SNARC was to build a data portal to store their existing datasets. During the process of building the data portal, I learned a lot about project management and geographic information system. In addition, my programming skill got improved.

This project helped me get a better cognition of the ecosystem of the geographic information system. To visualize the information of the datasets on the map, I learned ArcGIS JavaScript API. When the project started, I stored the fishnet information in the ArcGIS Online web map and displayed the whole web map in the data portal. When the user uploaded the new data, the system needs to update the attribute table to synchronize 
the data between the ArcGIS online and the local relational database. However, users can also edit and delete the datasets in the data portal; it is hard to adjust the web map at the same time. Another problem of using the ArcGIS Online web map is that my client must get an ESRI account, and this will add the unnecessary dependencies to the project. So, the final solution to the problem is to use another API to generate the map on the fly. When the user opens a map page in their browser, the system will generate a web map and several layers with the parameters in the API were called. The parameters included the fishnet and dataset geometries information. This method avoided the synchronize the data and reduce the dependencies of the system.

At the very beginning, this project was built with the Flask web platform. Flask is a micro Python web platform. After the first demo to the client, the manager in SNARC mentioned that they need the user and permission system in the data portal. This function was not available in the Flask, so I switched the platform to Django. The user management system was built in the Django, and I customed some functions in the group feature as the permission system. This is the first time I customized the source code of the open-source software. With this project, my coding skill got a significant improvement.

\subsection{Summary}

Collect the datasets with the data portal can help the researchers in SNARC design more analysis tools and extract more information from the data. The sample shown in this chapter is just a beginning; the future work about the analysis tool can help the leader in SNARC to make decisions. The next chapter is a summary of this project. 



\section{Chapter 7 - Conclusions and Future Work}

One web portal was developed to solve four problems addressed in this project. Section 7.1 presents a summary of this report. With the amount of data raising in this project, more future work can add to this project. Section 7.2 introduces a hydrological analysis system design.

\subsection{Summary and Conclusion}

Three significant problems are presented in chapter one; the goal of this project is to build a web portal to help the client collect, store, index, and share the dataset in their organization. This project built a web application and several tools with Python and ArcGIS to solve those problems. The ArcGIS API was used in this project to generate the map on-fly and display the map on the web page. This project also followed the decoupling design to store critical data locally in the JSON file or relational database. Only the public ArcGIS API was used in this project, so the client does not have to maintain an ArcGIS account.

This project is followed the process of product management during processing. Considering the deliverable of this project is a web application, the agile software development method was used in the developing process. The client can review the new feature regularly that prevent lots of misunderstanding.

\subsection{Hydrological Analysis System}

The metadata of the datasets in the data portal is all completed because of all the dataset that are uploaded to the data portal should pass the metadata validation check tool. The system can analyze some hydrological features with the metadata from the datasets and the river flowing maps.

The first step is to find which water body the dataset belongs to. This problem can be solved with the lake boundaries layer and intersect geoprocessing tool. The process of the matching the lake and dataset can be run in the backend after the dataset is uploaded. The intersect tool is the primary method in the tag analysis tool. Researcher can use the watershed number to query all the lakes in this area and run the intersect tool for each one to get the result. After that query, the lake name in the lake and water flowline relation map can be used to find the water flowline directly connected with this lake. The name of the lake and id of flowline will be the new tag for this dataset. 


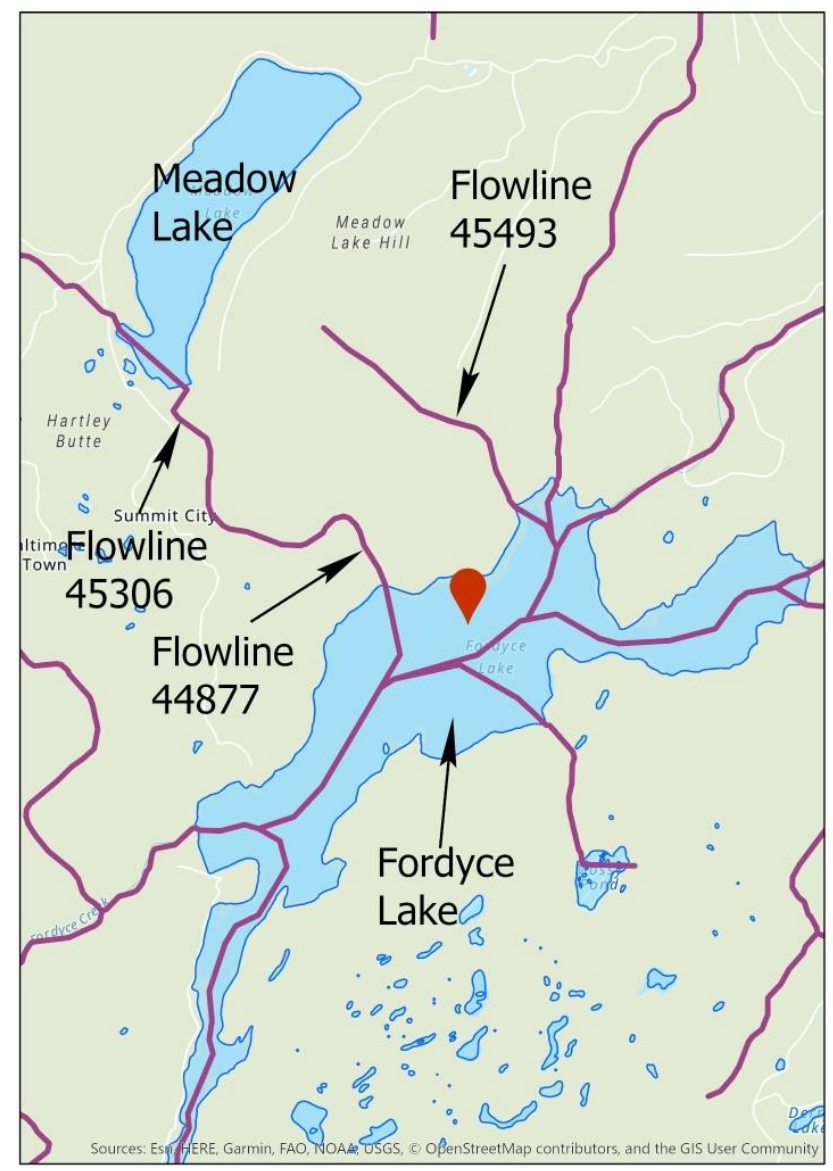

\section{Sample of Hydrological Tool}

Dataset location

Tag from uploader:

Microorganism, Hydrobiology

Tag from analysis tool:

Fordyce Lake, Flowline 44877, Flowline 45493 ...

Tag from Hydrological tool:

Meadow Lake, Mossy Pond,

Flowline 45306

Figure 6-2: Principle of Hydrological Analysis Tool

The second step is running the hydrological analysis tool to find more connections base on the tag in the last step. The theory of this step is to query the tag from the last step in the lake and water flowline relation map to get more hydrological relationships for the dataset. This step can be recursive for multiple times to get more results. The researchers can set up recursive times based on their usage. More recursive means more system resources used. Figure 6-2 shows the result of the whole process. The tag Fordyce Lake is from the tag analysis tool because the dataset's geographic location intersects with the Fordyce Lake boundary. The flowline number in the tag means those flowlines directly connected with the Fordyce Lake. In this case, the recursive time in the hydrological tool is one, so it adds the Meadow Lake and Mossy Pond that connects with the one flowline with the Fordyce lake. 


\section{Works Cited}

Bayer, I., \& McCreight, E. R. (1970). Organization and maintenance of large ordered indices. In. Proc. ACMSIGFIDET Workshop on Data Description and Access, Houston, Texas, Nov.

Desjardins, J. (2019, April 15). How Much Data is Generated Each Day? Retrieved from Visual Capitalist: https://www.visualcapitalist.com/how-much-data-is-generatedeach-day/

Farquhar, F. P. (2007). History of the Sierra Nevada. Univ of California Press.

Frost, R. A. (1982). Binary-relational storage structures. The Computer Journal 25(3), 358-367.

Herbst, D. B., Silldorff, E. L., \& Cooper, S. D. (2009). The influence of introduced trout on the benthic communities of paired headwater streams in the Sierra Nevada of California. Freshwater Biology, 1324-1342.

Hill, M. (1975). Geology of the Sierra Nevada (No. 37). Univ of California Press.

Jenkins, T. M., Knapp, R. A., Kratz, K. W., \& Cooper, S. D. (1994). Aquatic biota in the Sierra Nevada: Current status and potential effects of acid deposition on populations. Final Report.

Knapp, R. A. (1996). Non-native trout in natural lakes of the Sierra Nevada: an analysis of their distribution and impacts on native aquatic biota. Sierra Nevada ecosystem project: final report to Congress, 363-407.

Knapp, R. A., Matthews, K. R., \& Sarnelle, O. (2001). Resistance and resilience of alpine lake fauna to fish introductions. Ecological Monographs, 401-421.

Lawson, A. C. (1921). The Sierra Nevada. University of California Chronicle, Volume 23, Issues 1-2, 130-149.

Leland, H. V., Fend, S. V., \& Mahood, A. D. (1986). Composition and abundance of periphyton and aquatic insects in a Sierra Nevada, California, stream. The Great Basin Naturalist, 595-611.

Maciolek, J. A. \& Tunzi, M. G. . (1968). Microseston Dynamics in a Simple Sierra Nevada Lake-Stream System. Ecological Society of America, 60-75.

Matthews, K. R., \& Knapp, R. A. (1999). A study of high mountain lake fish stocking effects in the US Sierra Nevada Wilderness. International Journal of Wilderness, 24-26.

Meissner, K., \& Muotka, T. (2006). The role of trout in stream food webs: integrating evidence from field surveys and experiments. 75(2), pp. 421-433.

Srinath, K. R. (2017). Python-The Fastest Growing Programming Language. International Research Journal of Engineering and Technology (IRJET), 4(12), 354-357.

Stroh, G., \& Shalit, M. (2015). A lightning multiple casualty incident in Sequoia and Kings Canyon national parks. Wilderness \& Environmental Medicine, 43-53. 
Viers, J. H. (2011). Freshwater conservation options for a changing climate in California's Sierra Nevada. Marine and Freshwater Research, 266-278.

Wilkie, J. A., \& Hering, J. G. (1998). Rapid oxidation of geothermal arsenic (III) in streamwaters of the eastern Sierra Nevada. Environmental science \& technology, 657-662. 


\section{Appendix A. SQL Database Design}

\begin{tabular}{|c|c|c|}
\hline Field name & Field Type & Description \\
\hline data_id & PK & The auto-increment primary key. \\
\hline title & varchar & The title of the dataset. \\
\hline data_url & varchar & $\begin{array}{l}\text { The URL of the dataset which can be used for } \\
\text { download. This URL is the link to Cloud Storage. }\end{array}$ \\
\hline latitude & varchar & The latitude of the dataset center point. \\
\hline longitude & varchar & The longitude of the dataset center point. \\
\hline south & float & The south location of the dataset. \\
\hline north & float & The north location of the dataset. \\
\hline east & float & The east location of the dataset. \\
\hline west & float & The west location of the dataset. \\
\hline method & varchar & The method of the dataset. \\
\hline abstract & varchar & The abstract of the dataset. \\
\hline note & varchar & The note of the dataset. \\
\hline begin_date & datafield & The beginning date of the data collection. \\
\hline end_date & datafield & The end date of the data collection. \\
\hline Fishnet_1 & integer & $\begin{array}{l}\text { The fishnet id of the fishnet } 1 \text {. Used to update the } \\
\text { attribute table. }\end{array}$ \\
\hline Fishnet_2 & integer & $\begin{array}{l}\text { The fishnet id of the fishnet } 2 \text {. Used to update the } \\
\text { attribute table. }\end{array}$ \\
\hline Fishnet_3 & integer & $\begin{array}{l}\text { The fishnet id of the fishnet } 3 \text {. Used to update the } \\
\text { attribute table. }\end{array}$ \\
\hline Fishnet_4 & integer & $\begin{array}{l}\text { The fishnet id of the fishnet } 4 \text {. Used to update the } \\
\text { attribute table. }\end{array}$ \\
\hline Fishnet_5 & integer & $\begin{array}{l}\text { The fishnet id of the fishnet } 5 \text {. Used to update the } \\
\text { attribute table. }\end{array}$ \\
\hline huc_4 & varchar & The 4 digits USGS watershed HUC id. \\
\hline huc_6 & varchar & The 6 digits USGS watershed HUC id. \\
\hline huc_8 & varchar & The 8 digits USGS watershed HUC id. \\
\hline huc_10 & varchar & The 10 digits USGS watershed HUC id. \\
\hline huc_12 & varchar & The 12 digits USGS watershed HUC id. \\
\hline file_location & fileField & $\begin{array}{l}\text { The temporary file upload location. Used to accept the } \\
\text { file from the Django form. }\end{array}$ \\
\hline uploader & FK & Foreign key. The id of the uploader. \\
\hline tags & $\begin{array}{l}\text { Many-to- } \\
\text { many }\end{array}$ & The many-to-many field with the table Tag. \\
\hline author & $\begin{array}{l}\text { Many-to- } \\
\text { many }\end{array}$ & The many-to-many field with the table Author. \\
\hline date_created & datetime & The time which this dataset first uploaded. \\
\hline
\end{tabular}




\begin{tabular}{|l|l|l|l}
\hline date_modified & datatime & The time in which this dataset data last updated.
\end{tabular}

Tag

\begin{tabular}{|l|l|l|}
\hline Field name & Field Type & Description \\
\hline id & PK & The auto-increment primary key. \\
\hline name & charfield & The name of the tag \\
\hline
\end{tabular}

Author

\begin{tabular}{|l|l|l|}
\hline Field name & Field Type & Description \\
\hline id & PK & The auto-increment primary key. \\
\hline first_name & charfield & The first name of the author. \\
\hline middle_name & charfield & The middle name of the author. \\
\hline last_name & charfield & The last name of the author. \\
\hline email & charfield & The email address of the author. \\
\hline role & charfield & The role of the author. \\
\hline user_id & FK & The foreign key. User id in the user table. \\
\hline
\end{tabular}

\section{Appendix B. Analysis tools}

Watershed tool main function

The function receive a location_data from the session and return the watershed id.

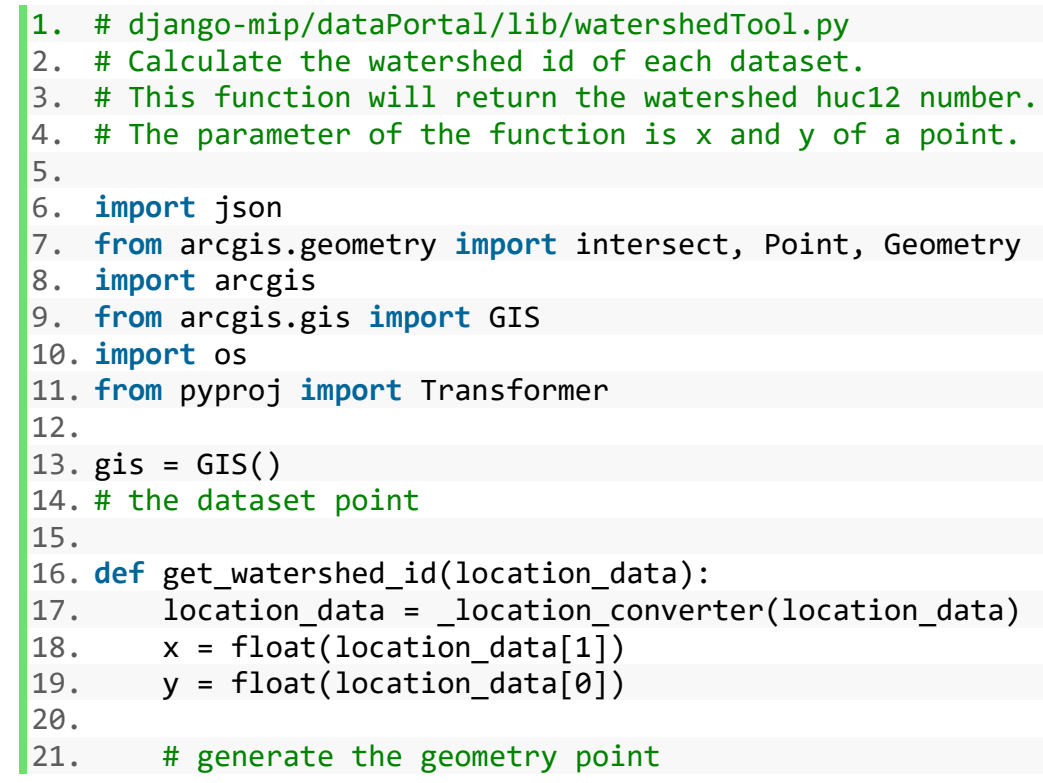




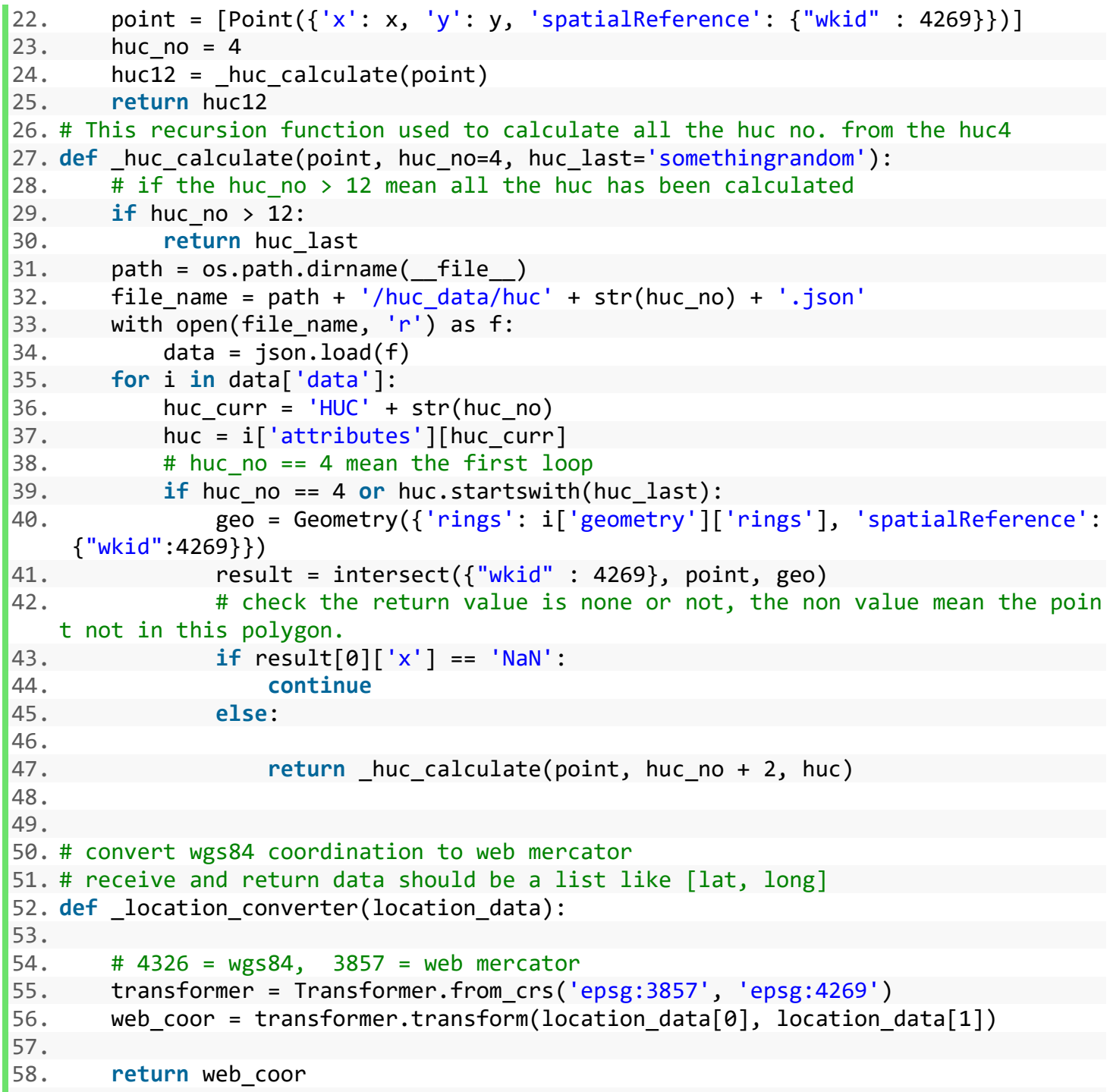

\section{Fishnet tool}

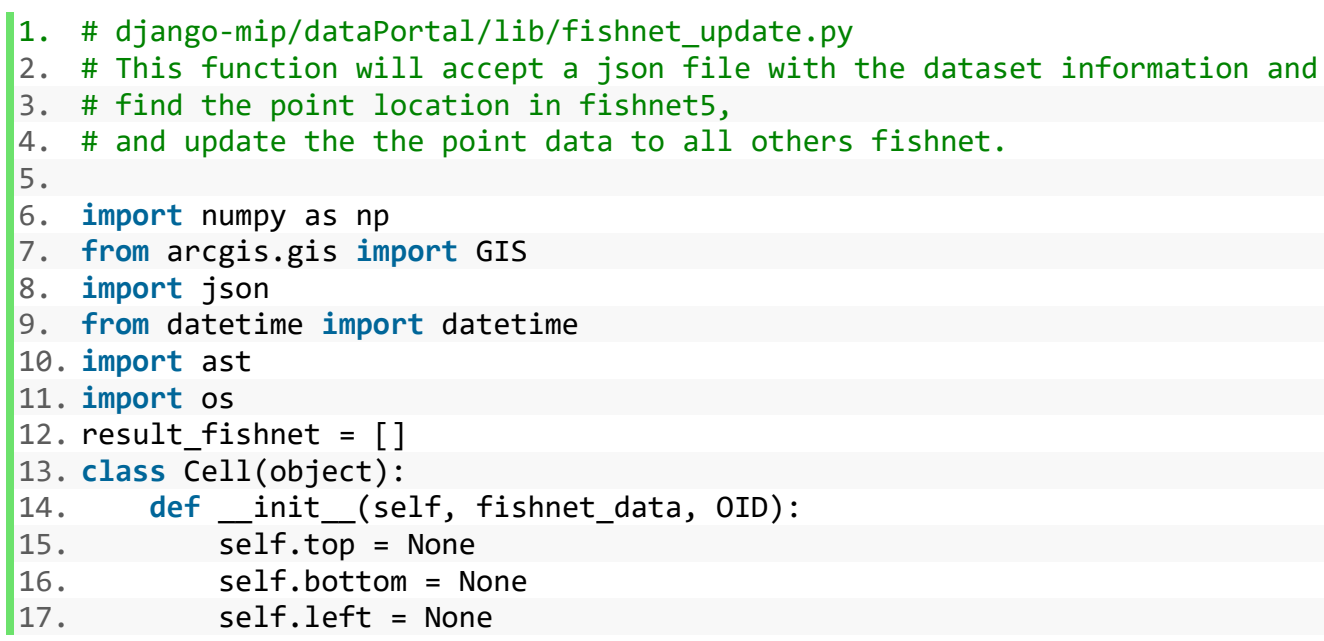




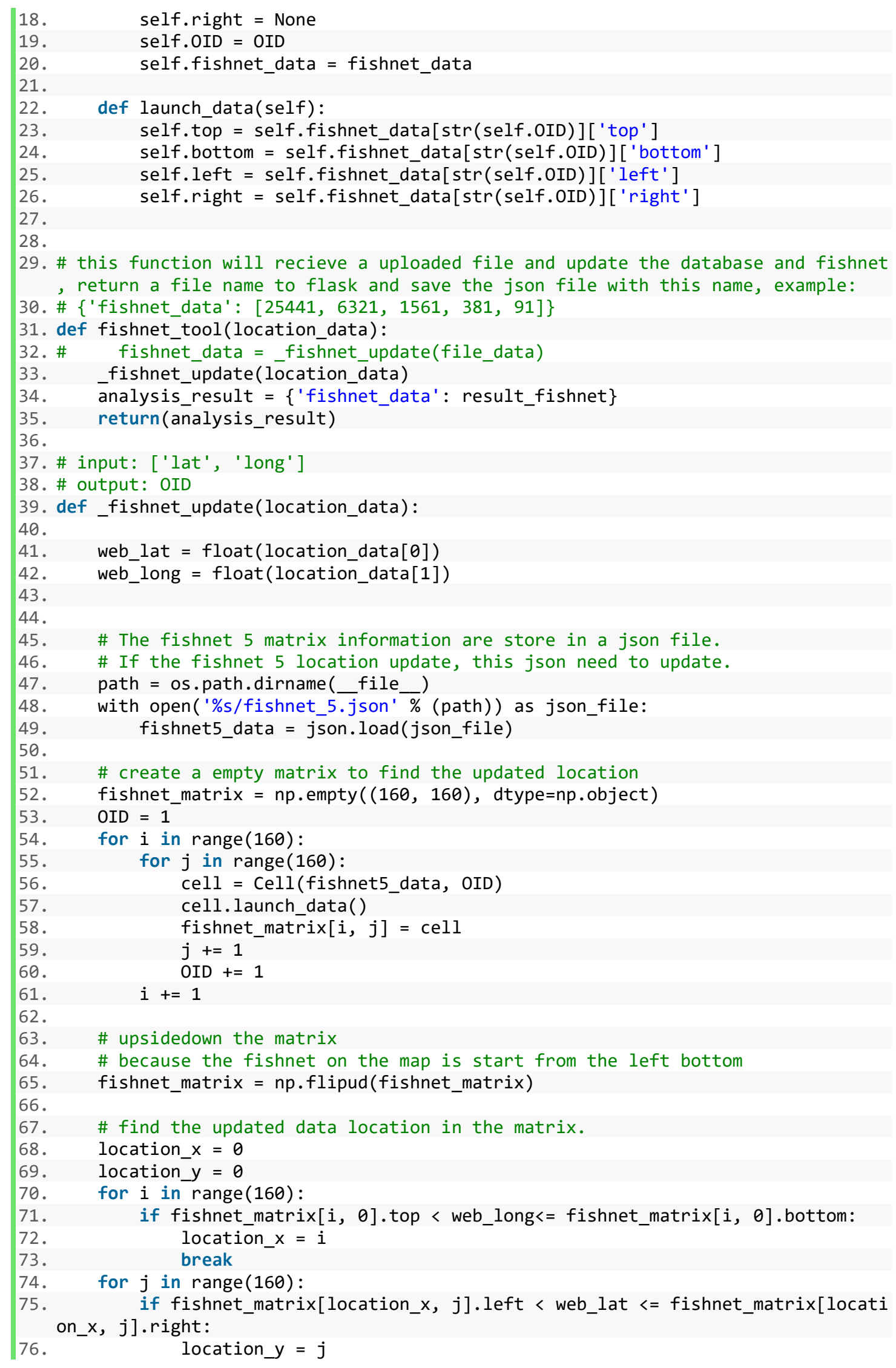




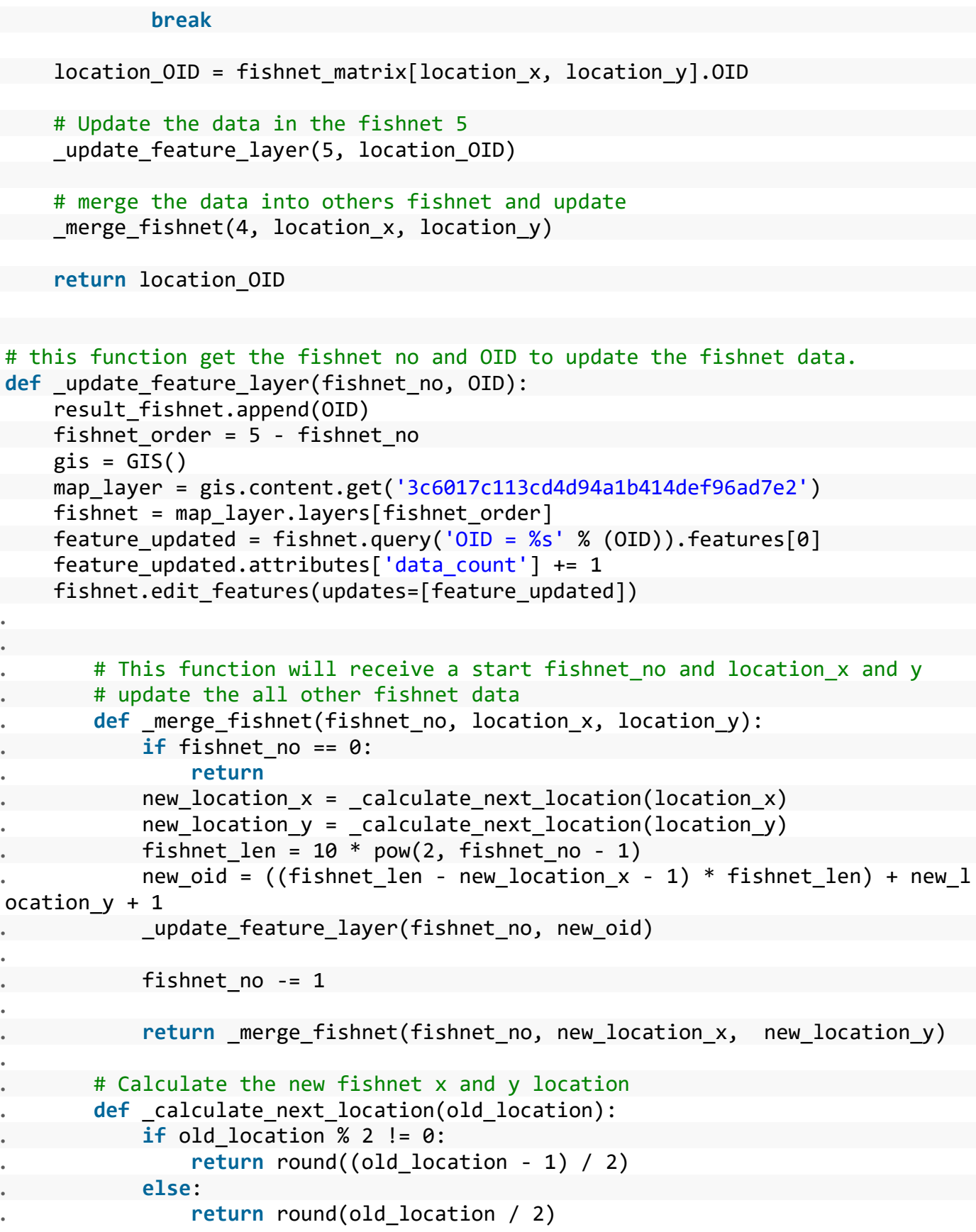

\section{Appendix C. Django}

The view.py control all the business logic of each page in Django.

1. \# django-mip/dataPortal/views.py

2. from django.shortcuts import render, redirect, get_object_or_404 


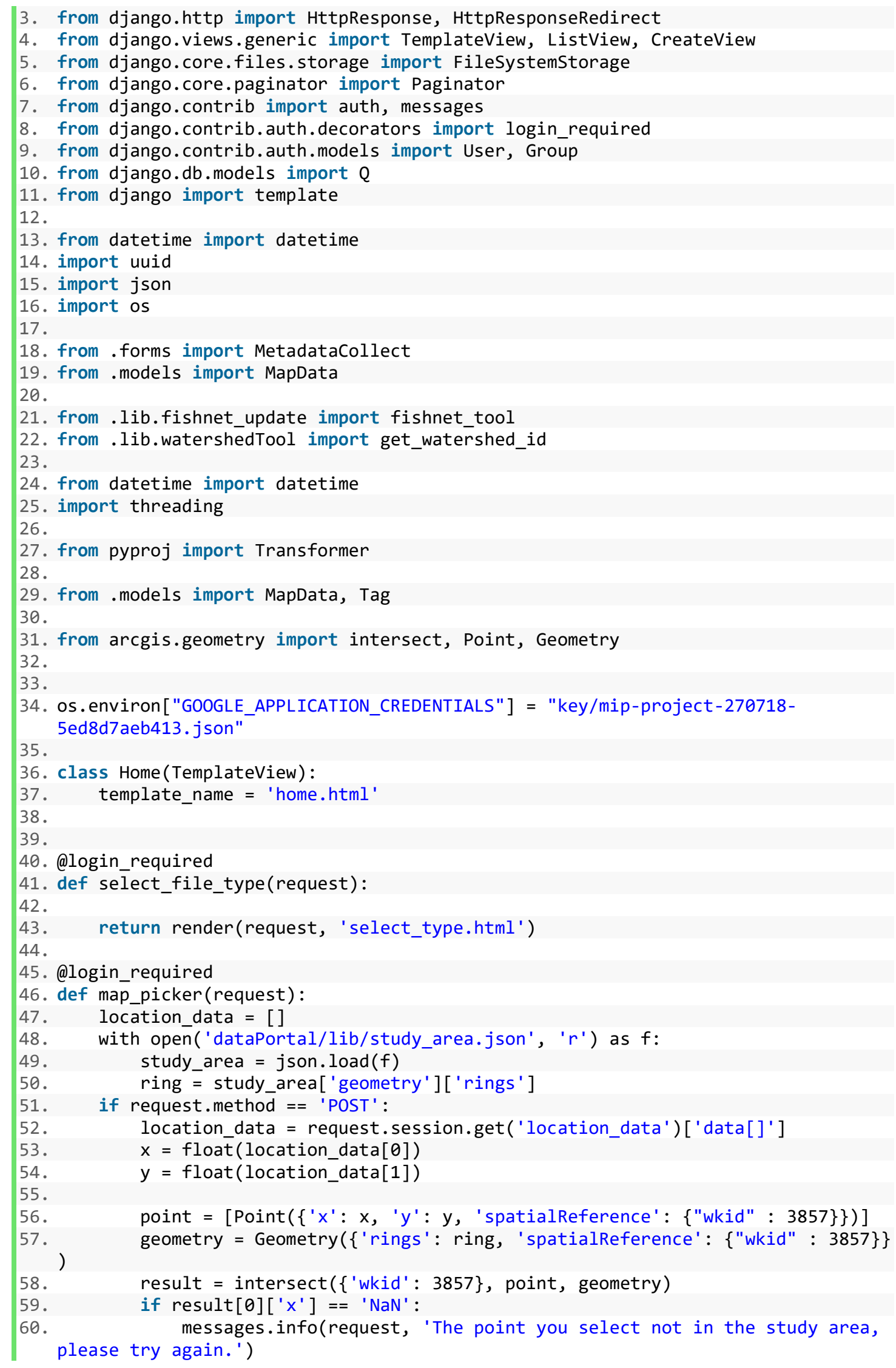




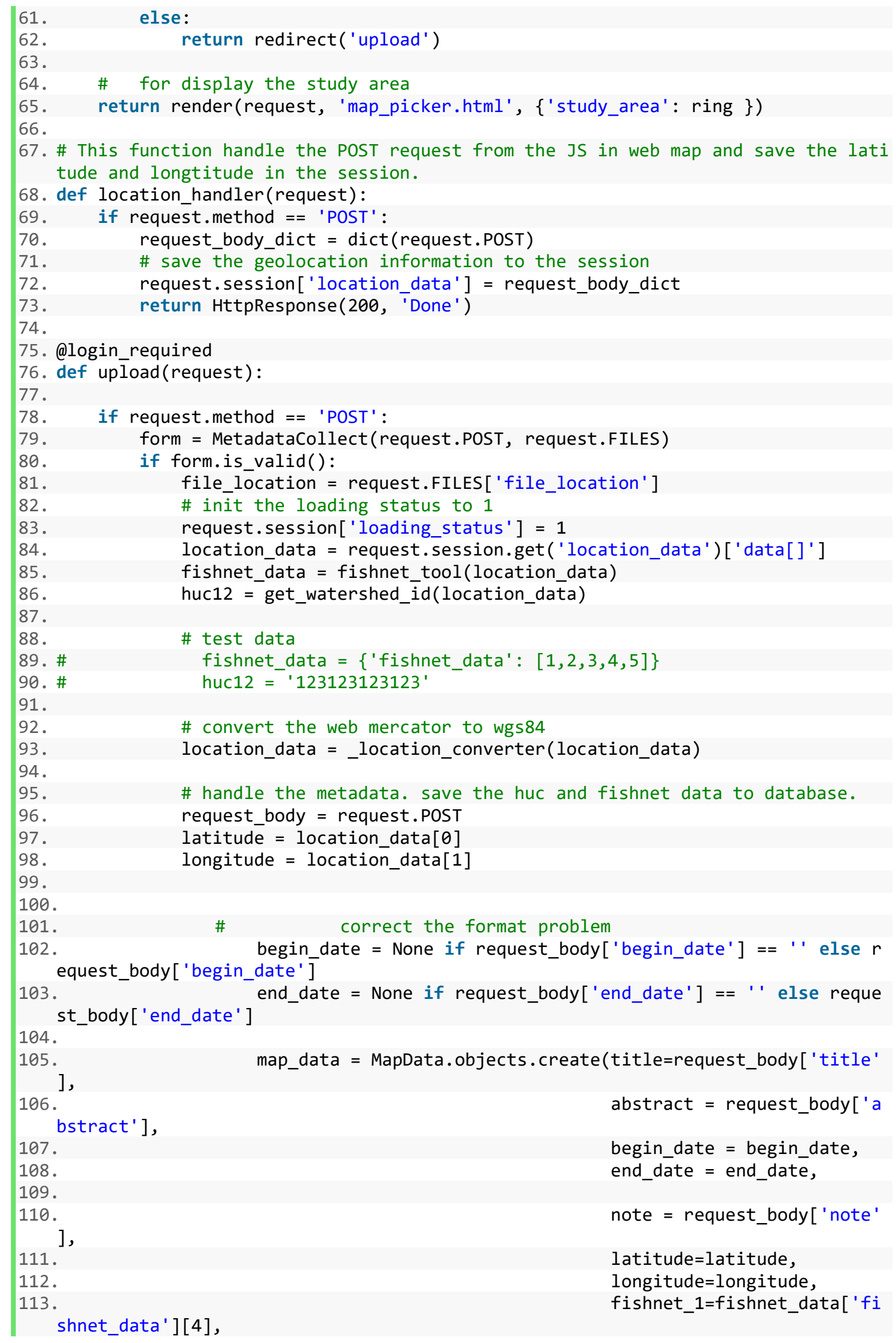




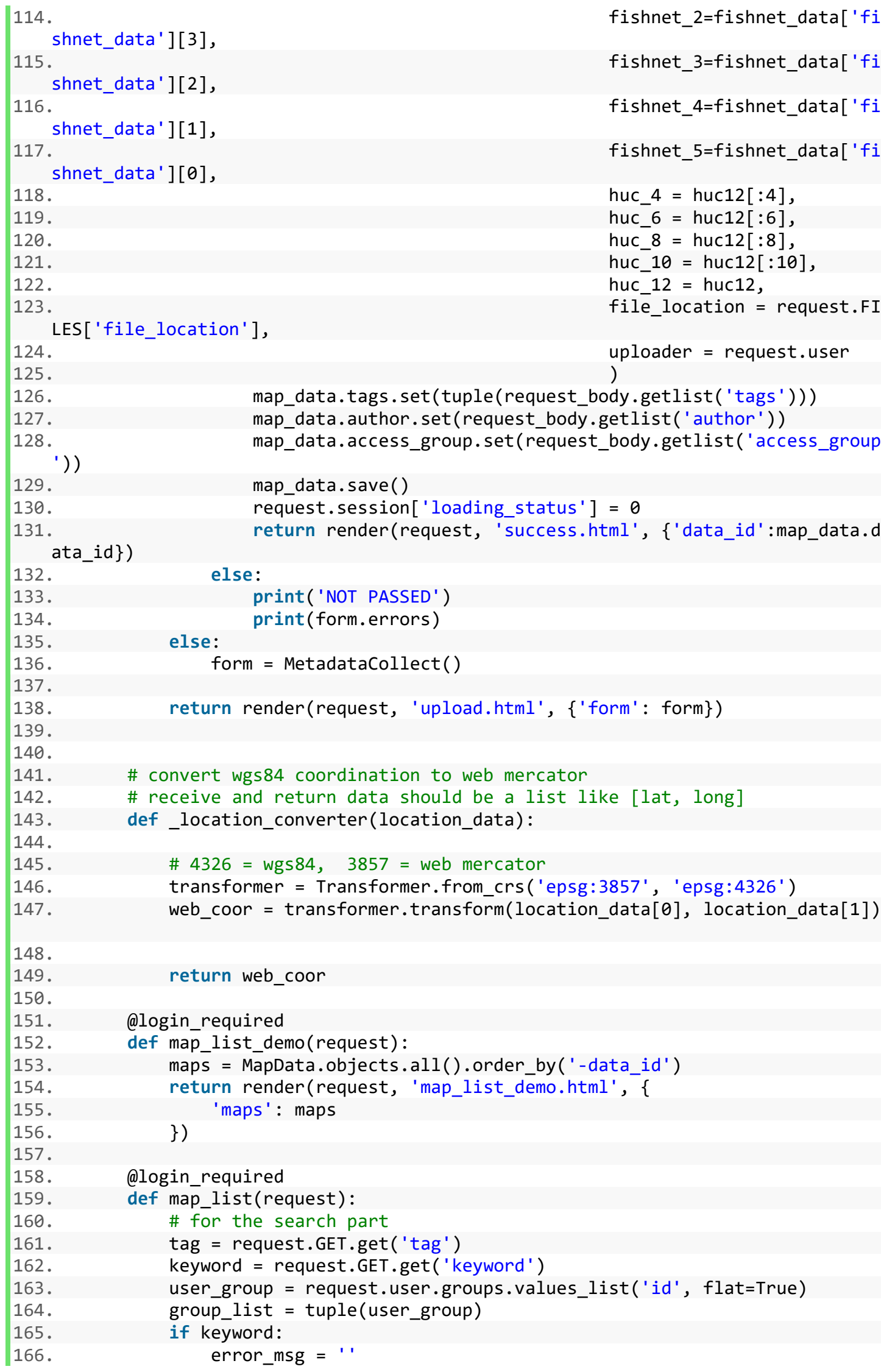




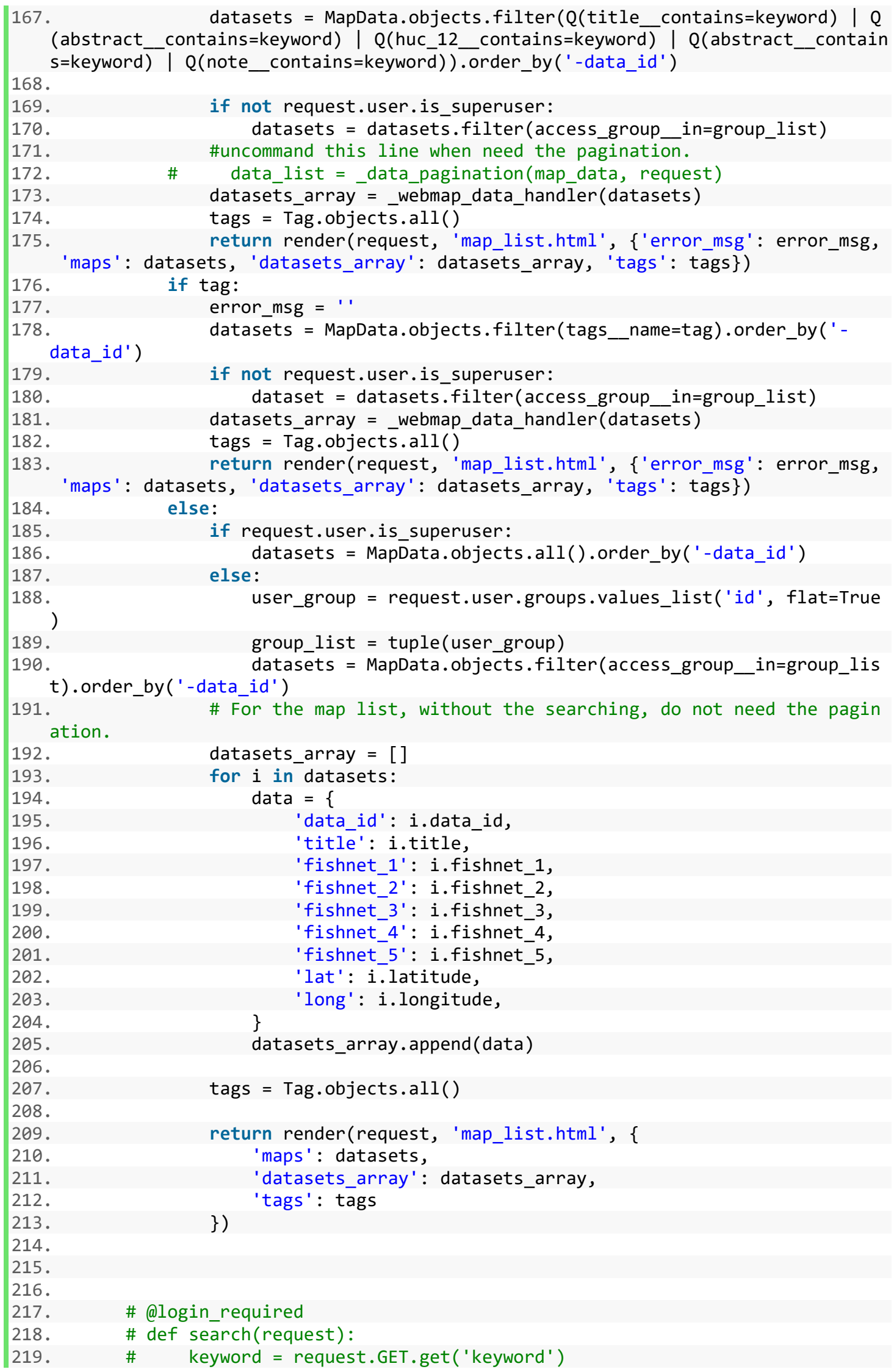




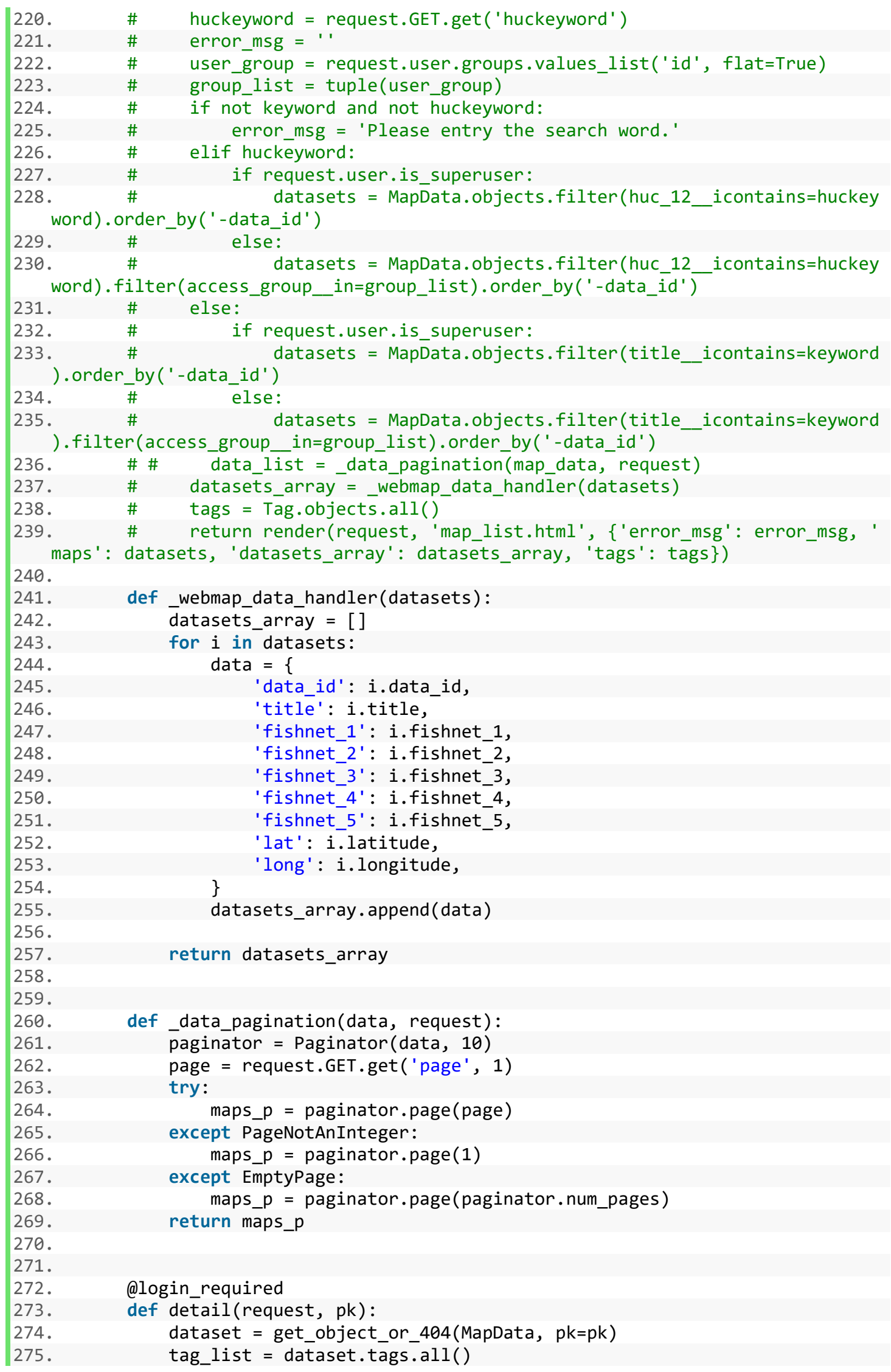




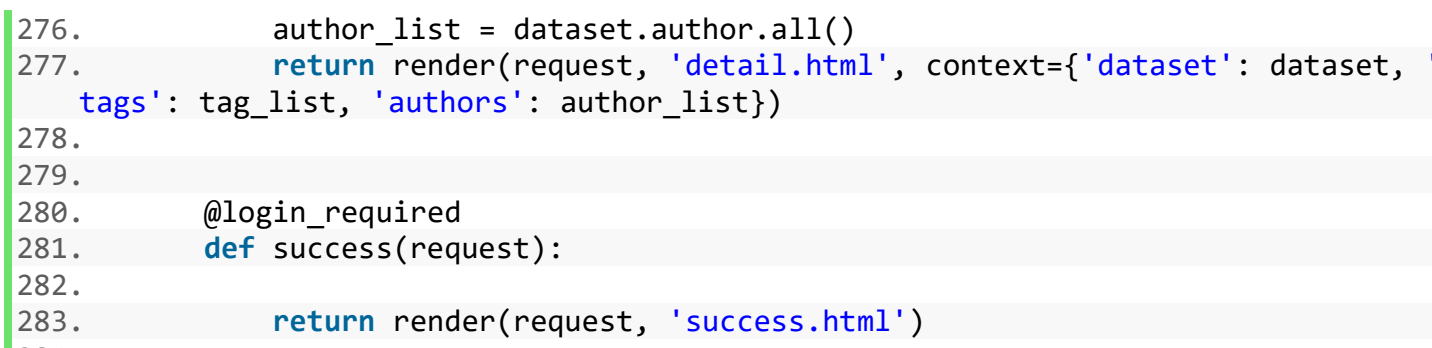

\section{Django database model}

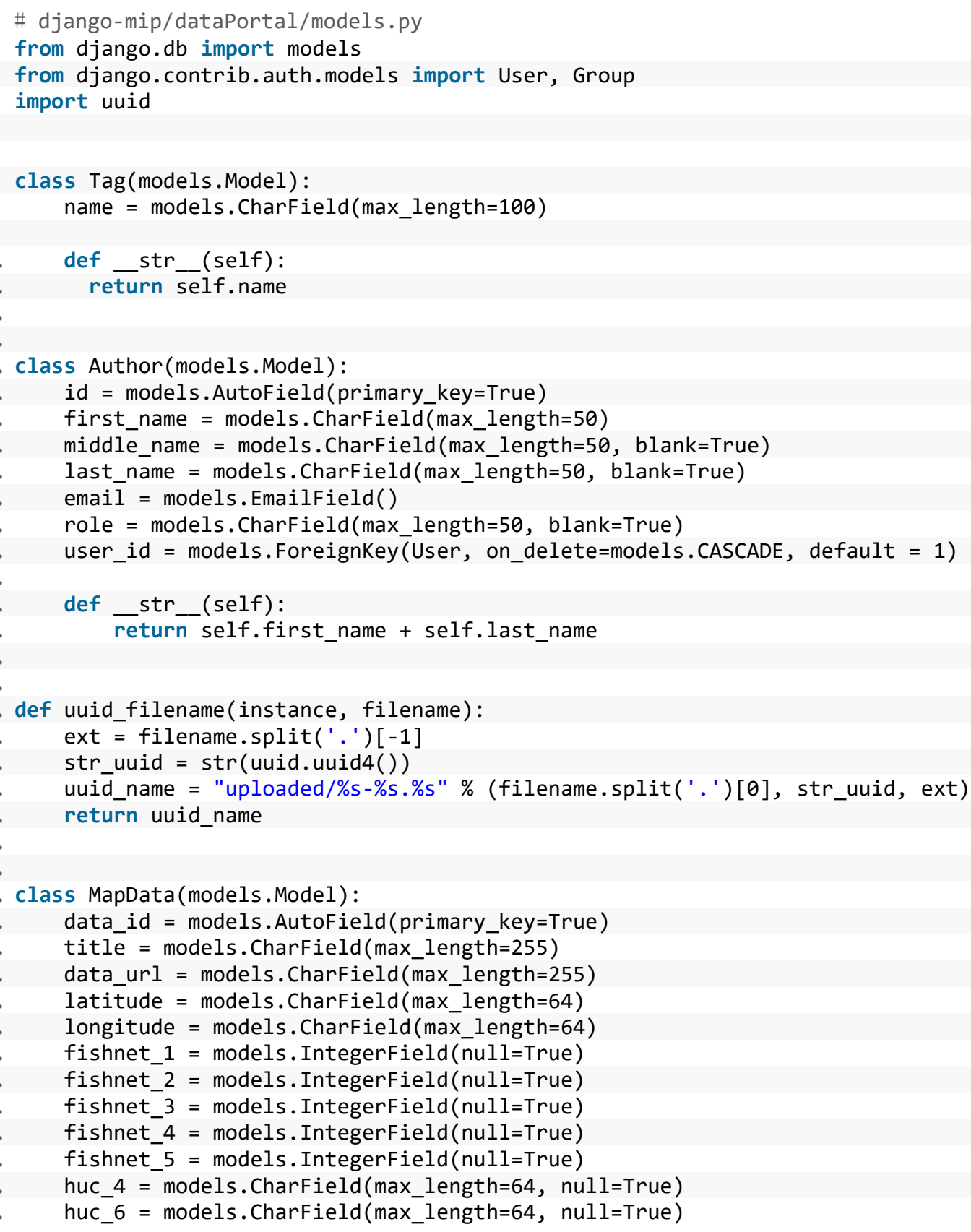




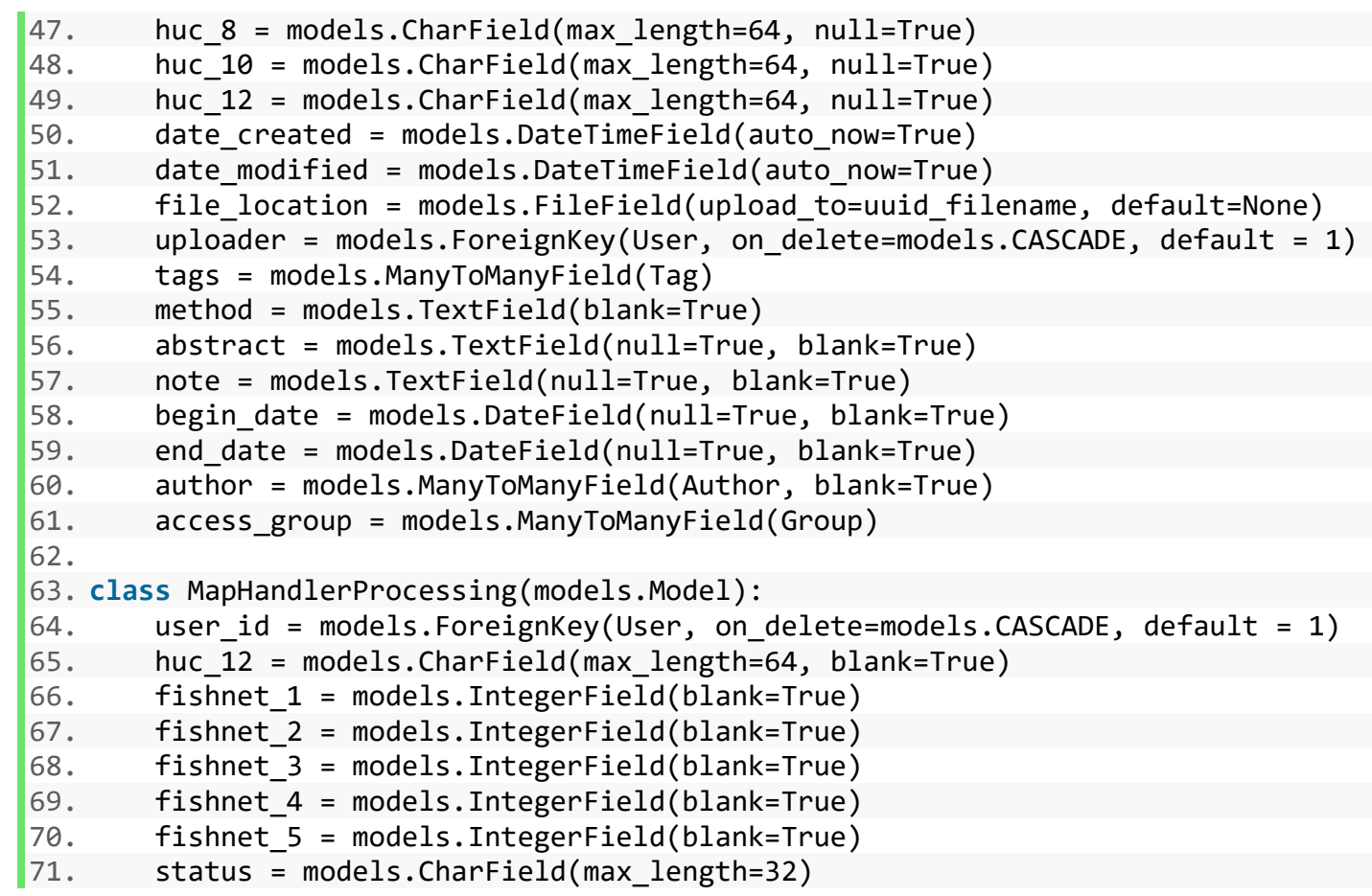

Map_list temple to display the dataset on the web map with the ArcGIS JavaScript API

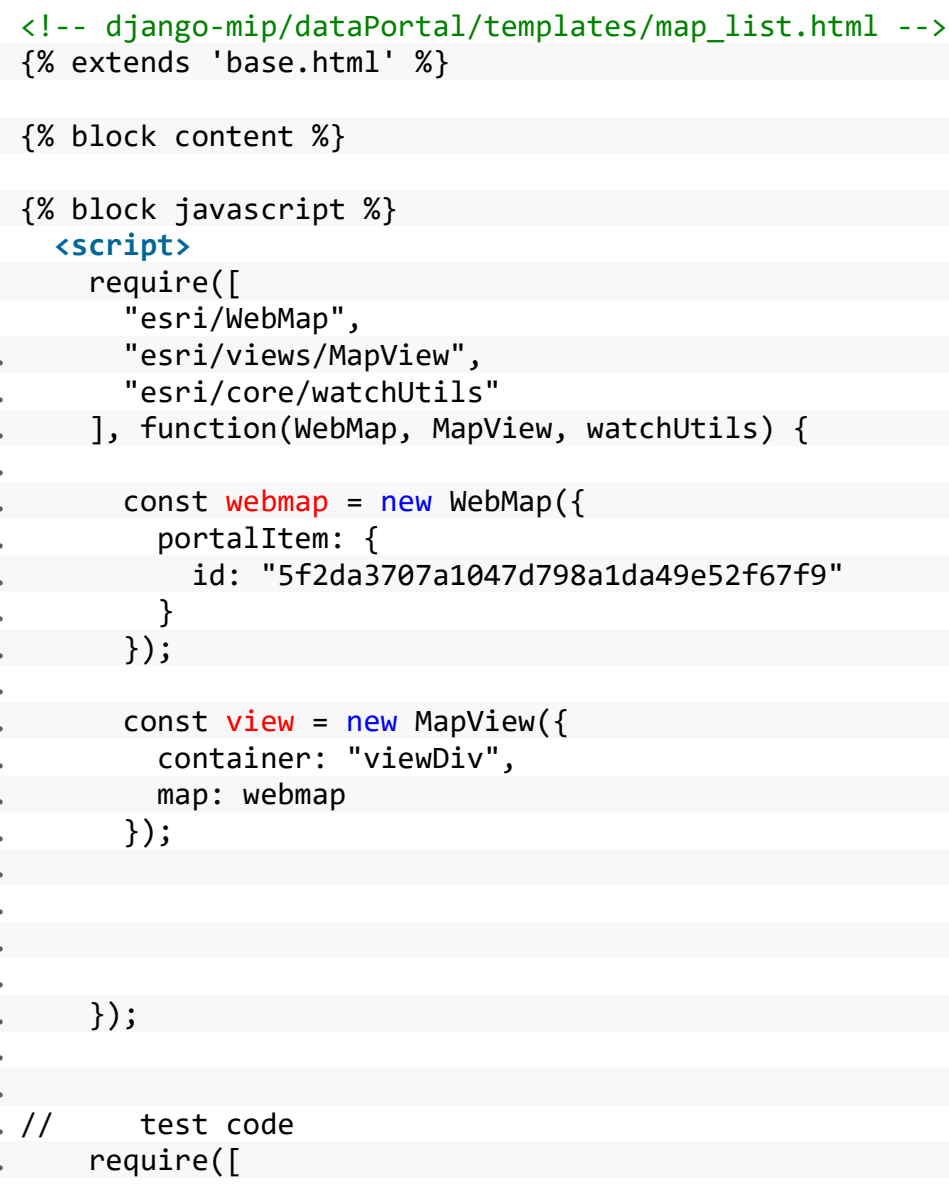




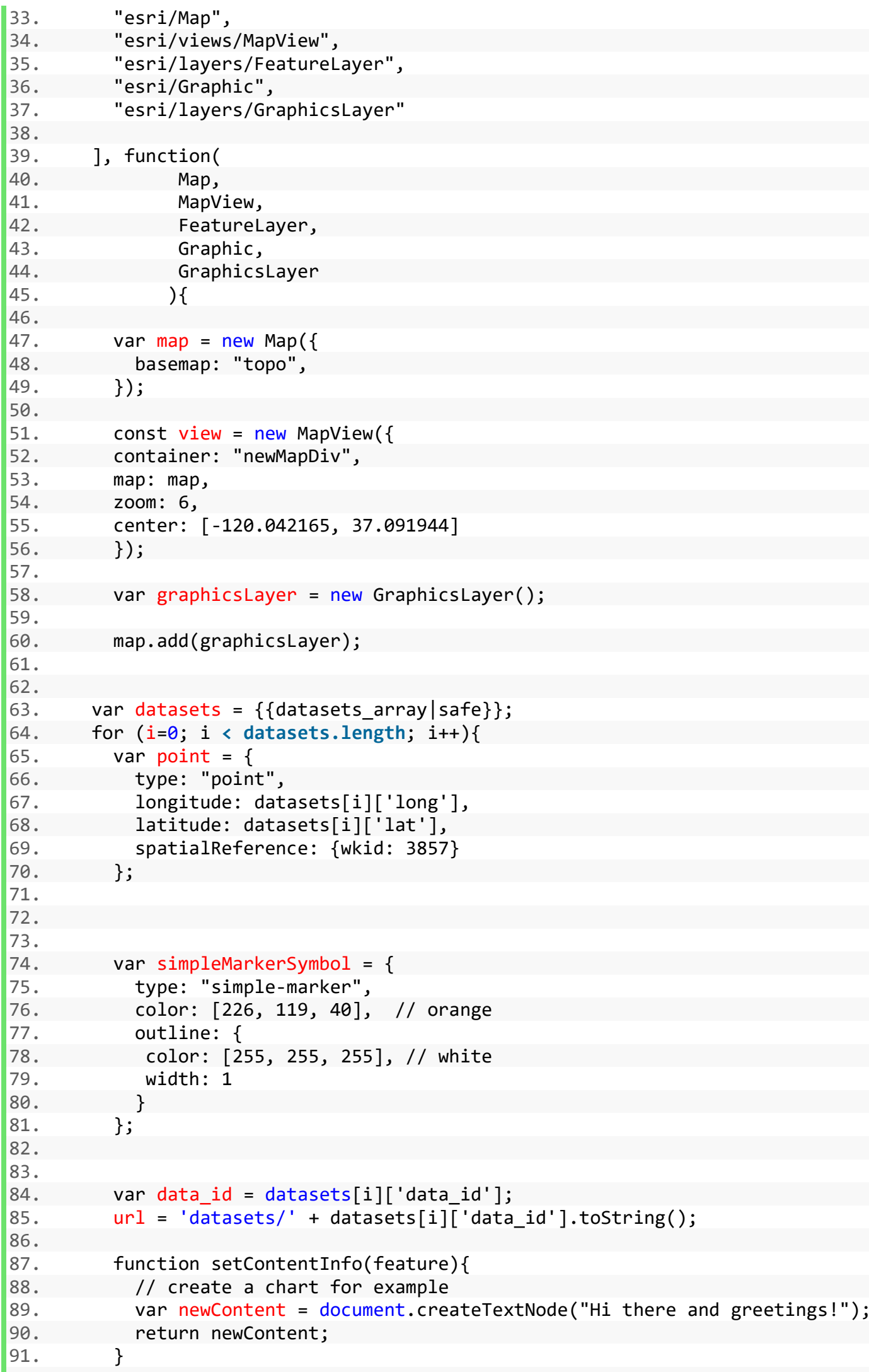




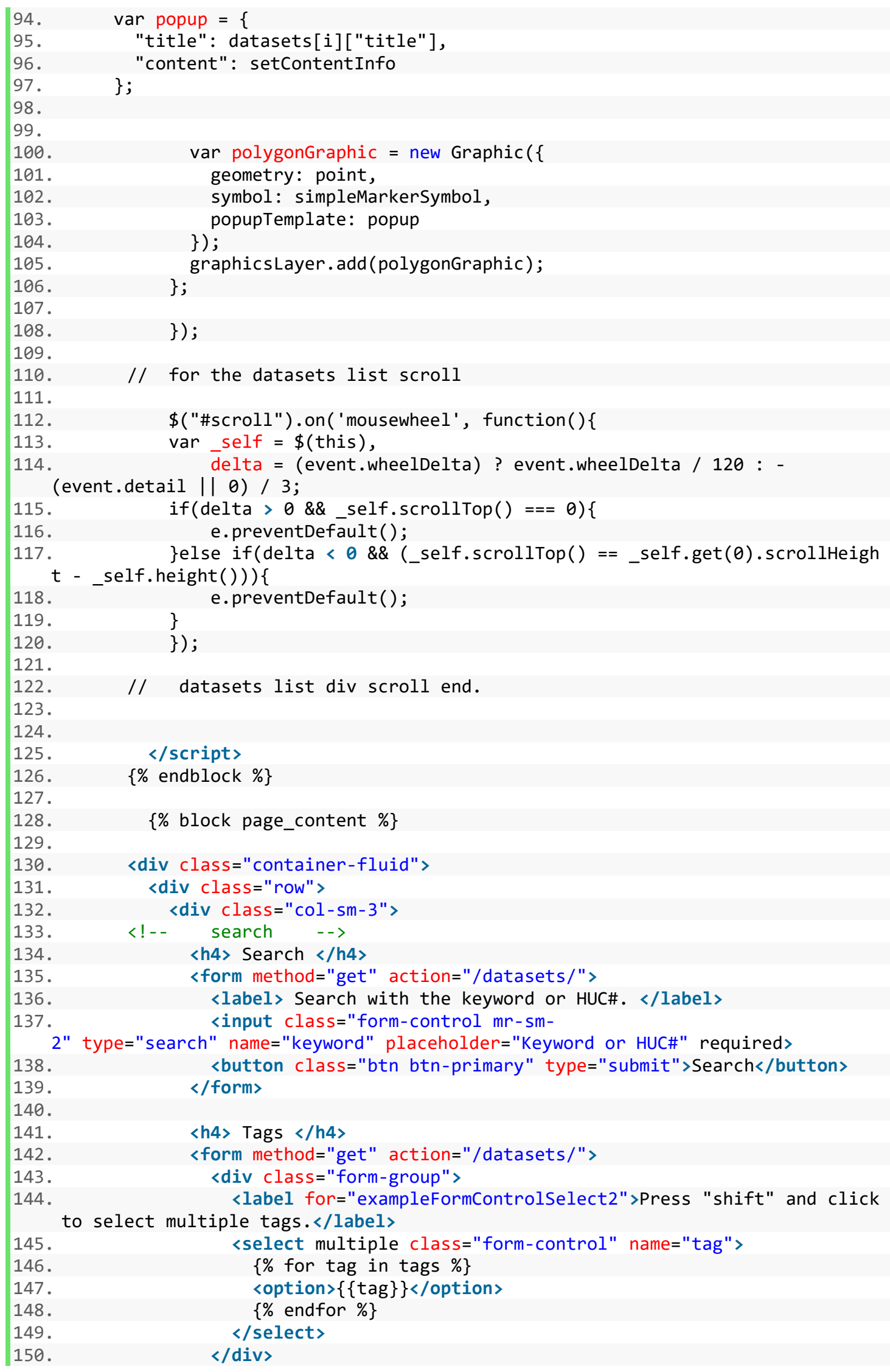




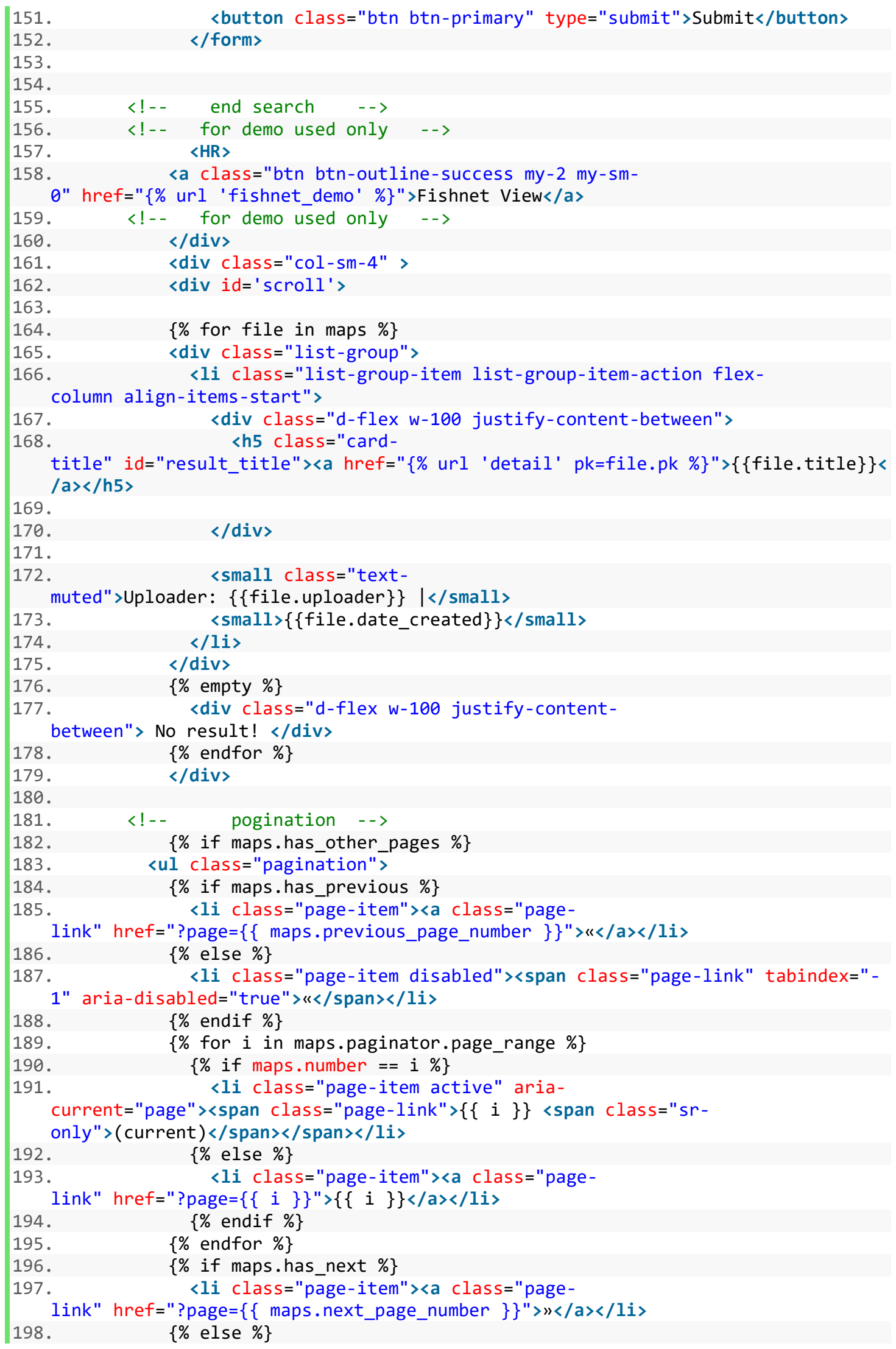




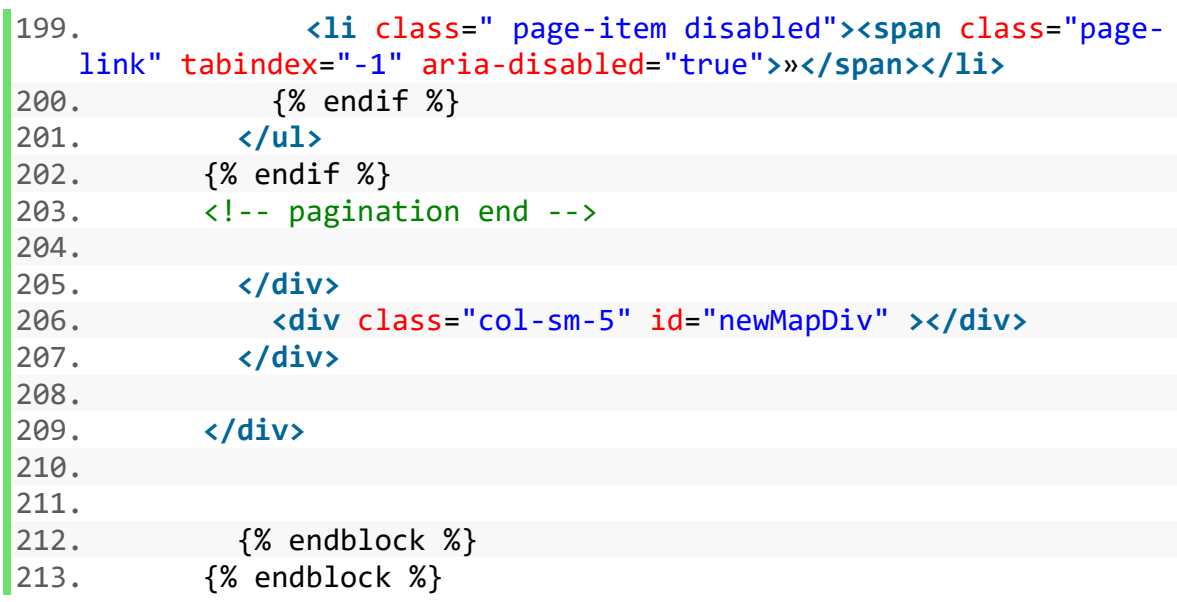

206.

207.

208.

209.

210.

211.

212. $\{\%$ endblock $\%\}$

213. $\{\%$ endblock \% $\}$ 